\title{
An Econometric Model of Network Formation With AN APPLiCATION TO BOARD INTERLOCKS BETWEEN FIRMS
}

\author{
Cristina Gualdani*
}

July 2019

\begin{abstract}
We study identification of the players' preferences in a network formation game featuring complete information, nonreciprocal links, and a spillover effect. We decompose the network formation game into local games such that the network formation game is in equilibrium if and only if each local game is in equilibrium. This decomposition helps us prove equilibrium existence, reduce the number of moment inequalities characterising the identified set, and simplify the calculation of the integrals entering those moment inequalities. The developed methodology is used to investigate Italian firms' incentives for having their executive directors sitting on competitors' boards.
\end{abstract}

KEYWORDS: network formation, multiple equilibria, partial identification, board interlocks.

JEL CODES: D85, C57, L22, G34.

\footnotetext{
${ }^{*}$ I gratefully acknowledge financial support from the European Research Council under the European Community's Seventh Framework Program FP7/2007-2013 grant agreement $N^{\circ}$ 295298. The views expressed in this paper are those of the author and do not involve the responsibility of the Bank of Italy. All errors are mine.

*Email: cristina.gualdani@tse-fr.eu, Toulouse School of Economics, University of Toulouse Capitole, Toulouse, France.
} 


\section{Introduction}

There is a successful literature showing that networks among individuals or organisations affect several outcomes of economic interest, such as academic achievement (Sacerdote, 2001), job search (Calvó-Armengol and Jackson, 2004), smoking decisions (Nakajima, 2007), criminal actions (Calvó-Armengol and Zenou, 2004), technology adoption (Conley and Udry, 2010), executives' compensation (Gayle, Golan, and Miller, 2015), and firms' performance (Cai and Szeidl, 2018). This recognition has fuelled a growing attention for estimating models in which agents purposefully create links according to a payoff configuration, an equilibrium notion, and an information structure (network formation models). Indeed, backing out agents' preferences for links is crucial because agents' link choices often change simultaneously with agents' behaviours on networks in response to policy interventions (Graham, 2015; de Paula, 2017).

Our paper considers a static game of network formation featuring complete information, nonreciprocal links, ${ }^{1}$ and a relatively small number of players. ${ }^{2}$ The payoff that player $i$ gets from linking to player $j$ is allowed to depend on the number of other players linking to $j$. Economic theory suggests that this spillover effect has a key role in many applications inspired by the corporate governance and industrial organisation literatures, e.g., board interlocks among competing firms (Thompson and McEwen, 1958; Allen, 1974; Mizruchi, 1996), hyperlinks among news websites (Dellarocas, Katona, and Rand, 2013), advice-seeking ties (Lazega, Lemercier, and Mounier, 2006; Lazega, et al., 2012; Lazega and Snijders, 2015), and trading connections (Kranton and Minehart, 2000a; 2000b; 2001).

The spillover effect considered implies that the network formation game admits multiple equilibria. Along the lines of the literature on entry games with multiple equilibria (Tamer, 2003; Galichon and Henry 2006; Ciliberto and Tamer, 2009; Beresteanu, Molchanov, and Molinari, 2011; Galichon and Henry, 2011; de Paula, 2013; Aradillas-Lopez and Rosen, 2016; Bontemps and Kumar, 2018), we provide partial identification arguments for the payoff parameters, under the assumption that the researcher has data on many equilibrium networks.

The methodological challenge faced is that econometric results from the literature on entry games with multiple equilibria cannot be directly applied to our setting. This is because they would lead to a characterisation of the sharp identified set based on a prohibitively enormous amount of moment inequalities, even with just 3 players. We overcome such an issue by leveraging on the structure assigned to the players' preferences. In particular, the spillover effect considered permits us to decompose the network formation game into some local games ${ }^{3}$ such that the network formation game is in equilibrium if and only if each local game is in equilibrium. The if and only if relation between the network formation game and the local games (hereafter, if and only if property) reveals that the set of equilibria of the network formation game is the Cartesian product of the set of equilibria of the local games, which thereby substantially decreases the curse of dimensionality in the analysis.

A principle analogous to the if and only if property is found in the study of single agent models when preferences are assumed separable in order to make difficult economic problems

\footnotetext{
${ }^{1}$ I.e., the existence of a link from agent $i$ to agent $j$ does not imply the existence of a link from $j$ to $i$.

${ }^{2}$ In practice, simulations suggest that our methodology works well for networks with up to 20 players.

${ }^{3} \mathrm{~A}$ local game of the network formation game is a game whose sets of players and strategy profiles are subsets of the network formation game's sets of players and strategy profiles.
} 
more manageable. In some sense, the if and only if property provides a reinterpretation of the separability notion in a context with interacting agents.

The if and only if property solves several problems hampering our ability to estimate the payoff parameters. First, it helps us prove the existence of an equilibrium of the network formation game, by building on results from Berry (1992). Second, it reduces the number of moment inequalities characterising the sharp identified set, by restricting the attention to the local games under some restrictions on the taste shocks and equilibrium selection. Third, it simplifies the calculation of the integrals which enter the moment inequalities, by showing that their value depend on the equilibria of the local games only. As a result, inference on the sharp identified set is computationally workable for sufficiently small networks. When networks are not sufficiently small, we also recommend an outer set of parameter values which seems to induce an almost unnoticeable loss of information with respect to the sharp identified set in simulations.

An illustration about inter-organisational ties demonstrates how the developed procedure works in practice. Most organisations are governed by a board of directors composed of executives and non-executives. The former lead the decision making process, the latter are involved in the supervision and guidance of the executives. In many European countries, firms often have some of their executives sitting on competitors' boards with or without executive duties. These links among companies are denominated primary horizontal board interlocks (hereafter, PHBIs). ${ }^{4}$ Deeply analysed by finance experts, PHBIs also draw the attention of economists because they might help firms transmit tacit knowledge, increase transparency, or encourage collusion, with a consequent impact on market structures. In this respect, the literature emphasises that firms could behave strategically when deciding whether to allow their executives to join rivals' boards. In particular, firms may prefer companies targeted also by other competitors, in order to promote meetings with as many industry actors as possible and, in turn, facilitate information flow (Thompson and McEwen, 1958; Allen, 1974; Mizruchi, 1996). Hence, firm $i$ 's payoff from having an executive sitting on rival $j$ 's board is expected to be increasing in the number of other competitors participating in $j$ 's board assemblies. Using the techniques discussed above, we are able to empirically test such a prediction. Specifically, we construct a $95 \%$ confidence region for the set of parameter values characterised in the first part of the paper using Italian data. Our results reveal that the spillover effect of interest has a positive sign, thus confirming that the creation of PHBIs is motivated by information sharing purposes. Lastly, via a counterfactual analysis, we use the estimated preferences to analyse the impact on networks and companies' payoffs of various policies restricting the formation of PHBIs, e.g., the enforcement of a law which prevents the biggest firms within each industry from creating PHBIs.

This paper borrows from two strands of the literature to provide a novel framework for studying identification in a network formation game. More precisely, in addition to the entry game literature cited earlier, this paper is connected to the literature on the econometrics of network formation models (Currarini, Jackson, and Pin, 2009; Christakis, et al., 2010; GoldsmithPinkham and Imbens, 2013; Leung, 2015; Boucher, 2017; Hsieh and Lee, 2016; Menzel, 2016;

\footnotetext{
${ }^{4}$ The adjective "horizontal" denotes the fact that firms are competitors (Carrington, 1981). The adjective "primary" denotes the fact that firms share directors with executive roles (Stokman, Van Der Knoop, and Wasseur, 1988).
} 
Miyauchi, 2016; Sheng, 2016; Badev, 2017; Mele, 2017; Ridder and Sheng, 2017; de Paula, Richards-Shubik, and Tamer, 2018; Leung, 2019). Miyauchi (2016) and Sheng (2016) are the most similar studies. Both works focus on reciprocal links ${ }^{5}$ and can handle a large number of players. The region of parameters values they characterise are neither shown to be sharp nor studied in relation to the sharp identified set. The spillover effects in Miyauchi (2016) are required to have a positive sign. The spillover effects in Sheng (2016) are assumed positive for some model specifications. Our methodology aims to be complementary to both papers. Indeed, it may be preferred by users who are interested in the spillover effect we analyse and work with small networks featuring nonreciprocal links. This is because our approach permits researchers to possibly exhaust all the implications of the model and data, and to test theoretical predictions on the sign of the spillover effect without restricting such a sign a priori.

This paper is also connected to the literature on inter-organisational ties, e.g., board interlocks, cross-ownerships, joint ventures, and supply and distribution channels. In particular, according to the inter-organizational linkage theories, companies are entities that possess interests. In pursuit of these interests, they form relations with other firms. For example, they share board members as an attempt to transfer information and, consequently, decrease investment uncertainty, anticipate disturbances, promote coordination, or convey expertise (Thompson and McEwen, 1958; Dooley, 1969; Allen, 1974; Pfeffer and Salancik, 1978; Aldrich, 1979), especially when this happens with competitors (Carrington, 1981; Leslie, 2004; Gabrielsen, Hjelmeng, and Sørgard, 2011; Waller, 2011) ${ }^{6}$ and through the exchange of executives (Mintz and Schwartz, 1981; Mizruchi, 1996; Mizruchi and Bunting, 1981; Stokman, Wasseur, and Elsas, 1985; Stokman, Van Der Knoop, and Wasseur, 1988; Mizruchi and Stearns, 1994). Most of the empirical findings on board interlocks focus on the correlation between a firm's profitability or size, and the intensity of board interlocks (Dooley, 1969; Pfeffer, 1972; Allen, 1974; Bunting, 1976; Pennings, 1980; Carrington, 1981; Burt, 1983; Meeusen and Cuyvers, 1985; Mizruchi and Stearns, 1988; Kaplan and Reishus, 1990; Booth and Deli, 1996). Some empirical works analyse the impact of board interlocks (exogenously taken or instrumented) on firms' internal decisions, e.g., executives' compensations (Hallock, 1997; Core, Holthausen, and Larcker, 1999; Patnam, 2013; Gayle, Golan, and Miller, 2015), patenting and R\&D spending (Helmers, Patnam, and Rau, 2015), and hiring choices (Lalanne, 2018). Our empirical illustration aims to shed further light on the role of board interlocks, by estimating, for the first time, a model in which firms' decisions about PHBIs are interdependent due to information sharing incentives.

In what follows, Section 2 illustrates the model, Section 3 develops identification arguments, Section 4 describes the empirical illustration, and Section 5 provides conclusions. Additional details are in the supplementary material. In terms of notation, bold case letters denote matrices, non-bold case letters denote vectors and scalars. Given the finite set $\mathscr{R},|\mathscr{R}|$ stands for $\mathscr{R}$ 's cardinality and $\mathscr{K}_{\mathscr{R}}$ indicates the collection of $\mathscr{R}$ 's non-empty subsets. Given $\mathscr{A} \subseteq \mathscr{R}, \mathscr{R} \backslash \mathscr{A}$ is the complement of $\mathscr{A}$ in $\mathscr{R}$. Lastly, given $\mathcal{N} \equiv\{1, \ldots, N\}, \mathcal{N}^{2}$ denotes the set of all the ordered pairs of different elements from $\mathcal{N}$.

\footnotetext{
${ }^{5}$ I.e., the existence of a link from agent $i$ to agent $j$ implies the existence of a link from $j$ to $i$.

${ }^{6}$ Also some policy reports, e.g., OECD (2008; 2010), discuss information exchanges between competitors through board interlocks.
} 


\section{A network formation game}

There are $N \in \mathbb{N} \backslash\{1,2\}$ players, labelled by the integers in $\mathcal{N} \equiv\{1, \ldots, N\}$, simultaneously deciding who to link with, under complete information. The players choose links in order to maximise payoffs which depend on the realised links and the players' characteristics.

We focus on frameworks featuring relatively small $N$. This is typical in applications inspired by the corporate governance or industrial organisation literature, as they are usually characterised by few key actors. For example, the empirical illustration in Section 4 has $N \leq 15$. We consider nonreciprocal links, such as PHBIs, trading connections, advice-seeking ties, and hyperlinks among websites. ${ }^{7}$

The output of the game is represented by an $N \times N$ matrix, $\boldsymbol{G}$, with the $i j$-th component, $G_{i j}$, equal to 1 if the link from player $i$ to player $j$ (hereafter, link $i j$ ) is formed and 0 otherwise. $\boldsymbol{G}$ is possibly asymmetric to embed non-reciprocity of relationships, i.e., $G_{i j}$ can be different from $G_{j i}$ for any $(i, j) \in \mathcal{N}^{2}$. There are no self-links, i.e., $G_{i i}=0 \forall i \in \mathcal{N}$. $\boldsymbol{G}$ is often referred to as a directed network. The players are also denominated the nodes of the network. Links from player $i$ to the other players are $i$ 's outgoing links. Links from the other players to player $i$ are $i$ 's incoming links.

For simplicity of exposition and without loss of generality, we present the methodological part of the paper by focusing on settings where the formation of the link $i j$ requires the consent of player $i$ only (hereafter, unilateral case). Examples are individuals nominating who they would ask for advice on technology adoption and hyperlinks among websites. Appendix B explains how all the results can be replicated in settings where the consent of both players $i$ and $j$ is necessary for forming the link $i j$ (hereafter, bilateral case), further differentiating between transferable and non-transferable payoffs. Examples are PHBIs, trading connections, and consulting ties.

More formally, in the unilateral case the players decide on forming outgoing links. For each player $i \in \mathcal{N}$, a pure strategy vector is $G_{i}$. $\equiv\left(G_{i j} \forall j \in \mathcal{N} \backslash\{i\}\right) \in\{0,1\}^{N-1}$. A pure strategy profile of the game is $\boldsymbol{G} \in \mathcal{G} \equiv\{0,1\}^{N(N-1)}$. Each player $i \in \mathcal{N}$ is endowed with some characteristics, $\left(X_{i}, \epsilon_{i}\right)$, assigned beforehand by nature. $X_{i} \in X \subseteq \mathbb{R}^{K}$ is observed by the researcher. $\epsilon_{i} \equiv\left(\epsilon_{i j} \forall j \in \mathcal{N} \backslash\{i\}\right) \in \mathbb{R}^{N-1}$ is unobserved by the researcher, where $\epsilon_{i j}$ represents player $i$ 's taste shock for the link $i j . \quad X \equiv\left(X_{i} \forall i \in \mathcal{N}\right)$ and $\epsilon \equiv\left(\epsilon_{i} \forall i \in \mathcal{N}\right)$ are common knowledge to all the players. The payoff that player $i$ gets from participating in the game depends on $(\boldsymbol{G}, \boldsymbol{X}, \epsilon)$ and is denoted by $u_{i}\left(\boldsymbol{G}, \boldsymbol{X}, \epsilon ; \theta_{u}\right)$, where $\theta_{u} \in \Theta_{u} \subset \mathbb{R}^{d_{u}}$ is a vector of parameters. The agents play pure strategy Nash equilibrium (hereafter, PSNE). ${ }^{8} \boldsymbol{G} \in \mathscr{G}$ is a PSNE if it is robust to multi-link deviations by each player.

Definition 1. (PSNE of the network formation game) $\boldsymbol{G} \in \mathscr{G}$ is a PSNE of the network formation game if

$$
U_{i}\left(G_{i}, \boldsymbol{G}_{-\{i \cdot\}}, \boldsymbol{X}, \epsilon ; \theta_{u}\right) \geq U_{i}\left(\tilde{G}_{i \cdot}, \boldsymbol{G}_{-\{i \cdot\}}, \boldsymbol{X}, \epsilon ; \theta_{u}\right),
$$

\footnotetext{
${ }^{7}$ Firm $i$ having an executive sitting on company $j$ 's board does not imply $j$ having an executive sitting on $i$ 's board. Agent $i$ buying from agent $j$ does not imply $j$ buying from $i$. Agent $i$ consulting agent $j$ does not imply $j$ consulting $i$. Website $i$ containing an hyperlink to website $j$ does not imply $j$ containing an hyperlink to $i$.

${ }^{8}$ As in Mele (2017), we use PSNE for the unilateral case. Instead, when analysing the bilateral case in Appendix B, we impose pairwise stability, which is more appropriate for situations where the formation of the link $i j$ requires the consent of both players $i$ and $j$. See, e.g., Bloch and Jackson (2006) for a discussion of various equilibrium notions in network formation games.
} 
$\forall \tilde{G}_{i} . \in\{0,1\}^{N-1} \backslash\left\{G_{i} \cdot\right\}$ and $\forall i \in \mathcal{N}$, where $\boldsymbol{G}_{-\{i \cdot\}}$ denotes the network $\boldsymbol{G}$ without the vector $G_{i}$.

\section{Identification}

We assume that the researcher observes the equilibrium network, $\boldsymbol{G}$, and the players' characteristics, $\boldsymbol{X}$, for many replications of the game (many-network setting). ${ }^{9}$ This section examines how to identify $\theta_{u}$ using those data.

\subsection{Assumptions on payoffs}

In Section 2 player $i$ 's payoff is in principle allowed to depend on the link decisions of all the other players. For example, the payoff that player $i$ gets from linking to player $j$ may depend on $i$ and $j$ 's links, the links that $i$ and $j$ have in common, the connections of the players linked with $j$, etc. In the network formation literature, such interdependences among the players' link decisions are referred to as spillover effects.

Spillover effects complicate the identification and estimation of $\theta_{u}$ for two reasons: first, they may cause non-existence of an equilibrium and make the model incoherent; second, they may generate multiple equilibria and make the model incomplete (Tamer, 2003). Multiple equilibria usually lead to partially identified parameters. Inference on the sharp identified set involves computing the sample analogues of an overwhelming amount of moment inequalities. Moreover, obtaining those sample analogues requires calculating integrals which depend on the set of equilibria of the network formation game. The integral calculation is done by simulation: we draw many realisations of the preference shocks, we enumerate all the possible networks, and we verify whether each candidate network is an equilibrium for every drawn realisation of the latent variables. Overall, implementing such a procedure for the general network formation game of Section 2 is computationally unworkable without further assumptions, even for $N=3$.

To improve tractability, researchers typically restrict the types of spillover effects considered. Our paper focuses on a specific spillover effect: the payoff that player $i$ gets from forming the link $i j$ is allowed to depend on the number of other players linking to $j$. Economic theory suggests that this spillover effect has a key role in many applications of interest. We provide four examples inspired by the corporate governance and industrial organisation literatures.

PHBIs As anticipated in Section 1, when firms decide whether to allow their executives to join rivals' boards, they may prefer companies targeted also by other competitors, in order to promote meetings with as many industry actors as possible and, in turn, facilitate information flow (Thompson and McEwen, 1958; Allen, 1974; Mizruchi, 1996). Hence, firm i's payoff from having an executive sitting on rival $j$ 's board is expected to depend on (and, specifically, be increasing in) the number of other competitors participating in $j$ 's board assemblies. More details on this application are in Section 4.

\footnotetext{
${ }^{9}$ The researcher is assumed to observe all the realised links. We abstract from statistical issues like missing data on the realised links or sampling data from the realised links, which are relevant instead in large network models.
} 
Hyperlinks among news websites Hyperlinks are extensively used by news websites to integrate their own content with more detailed information offered by a third party. An extreme case is represented by the so-called "content aggregators", such as Google News, Yahoo News, or the Huffington Post, which produce little original material and mostly refer to other news websites. Generally, the link source does not have to ask for permission or pay fees to the link target. In a world where news websites compete with each other to attract and monetise traffic, researchers want to learn about media's incentives behind such a unilateral free linking practice, in order to measure its impact on the production of high-quality content. In particular, on one hand, the link source reduces the time visitors spend at its site (and so sustains losses) by bringing them to the target. On the other hand, the link source gets revenues from the ability to attract new visitors by permitting access to the target. However, these revenues may suffer the rivalry of other news websites connecting to the same target (Dellarocas, Katona, and Rand, 2013). Therefore, news website $i$ 's payoff from linking to news website $j$ is expected to depend on (and, specifically, be decreasing in) the number of other news websites linking to $j$.

Advice-seeking ties Judges, attorneys, or consultants dealing with competition litigations typically face very heterogeneous cases whose resolution depends on the knowledge of the specific industry in which the conflict occurs. For this reason, they use each other for advice intensively. A careful analysis of the determinants of such advice-seeking ties is essential to explain the evolution of competition case law in various economic sectors. In particular, the literature emphasises that advice-seeking converges on recognised members of the community and that recognition is endogenous: members sought out by many agents build a reputation because selecting them is perceived as a safe source of legitimacy of expertise claims (Lazega, Lemercier, and Mounier, 2006; Lazega, et al., 2012; Lazega and Snijders, 2015). Therefore, agent $i$ 's payoff from asking agent $j$ for advice is expected to depend on (and, specifically, be increasing in) the number of other agents nominating $j$ as advisor.

Trading networks Trading networks consist of buyer-supplier ongoing contracts among firms and are commonly used by companies to exchange goods. They are distinguished from anonymous markets because a buyer can obtain a good from a seller only if these two are linked. Economists are interested in understanding whether the allocations generated by trading networks are efficient and aligned with social welfare. In particular, the literature points out that, given the necessity of forming a link in order to enjoy commerce in the future, the structure of ties may influence the competition for goods. This is because third parties could affect the agreement terms in bilateral transactions by changing the value of outside options (Kranton and Minehart, 2000a; 2000b; 2001). For example, consider a group of companies each offering a different service, e.g., legal, accounting, consulting, marketing, etc. Suppose that every company has to choose between internalising and buying from other firms in the group any service needed for operational activities. In light of the previous remarks, we expect a buyer's bargaining power with a seller to depend on (and, specifically, be decreasing in) the number of other companies buying from that seller.

Assumption 1 formalises the restrictions on the players' payoffs illustrated above. 
Assumption 1. (Spillover effect)

a) $u_{i}\left(\cdot ; \theta_{u}\right)$ is additively separable in player $i$ 's outgoing links, i.e.,

$$
u_{i}\left(\boldsymbol{G}, \boldsymbol{X}, \epsilon ; \theta_{u}\right) \equiv \sum_{j \in \mathcal{N} \backslash\{i\}} G_{i j} \times u_{i j}\left(\boldsymbol{G}_{-\{i \cdot\}}, \boldsymbol{X}, \epsilon ; \theta_{u}\right)
$$

where $u_{i j}\left(\cdot ; \theta_{u}\right)$ is the payoff that $i$ gets from forming the link $i j$.

b) $u_{i j}\left(\cdot ; \theta_{u}\right)$ is additively separable in $(\boldsymbol{X}, \epsilon)$ and $\boldsymbol{G}_{-\{i \cdot\}}$. Moreover, $u_{i j}\left(\cdot ; \theta_{u}\right)$ depends on $\boldsymbol{G}_{-\{i \cdot\}}$ only through the number of player $j$ 's incoming links and this dependence is monotonic. Specifically,

$$
u_{i j}\left(\boldsymbol{G}_{-\{i \cdot\}}, \boldsymbol{X}, \epsilon ; \theta_{u}\right) \equiv z_{i j}(\boldsymbol{X}, \epsilon ; \beta)+v_{j}\left(\sum_{k \in \mathcal{N} \backslash\{i\}} G_{k j} ; \delta\right),
$$

where $\beta$ and $\delta$ are vectors of parameters, $\theta_{u} \equiv(\beta, \delta)$, and $v_{j}(\cdot ; \delta)$ is monotone in $\sum_{k \in \mathcal{N} \backslash\{i\}} G_{k j}$.

For example, the following utility specification satisfies Assumption 1 for $N=4$ :

$$
\begin{aligned}
u_{i}\left(\boldsymbol{G}, \boldsymbol{X}, \epsilon ; \theta_{u}\right) \equiv & G_{i j} \times\left[\beta^{\prime}\left|X_{i}-X_{j}\right|+\delta \log \left(G_{h j}+G_{k j}+1\right)+\epsilon_{i j}\right]+ \\
& G_{i h} \times\left[\beta^{\prime}\left|X_{i}-X_{h}\right|+\delta \log \left(G_{j h}+G_{k h}+1\right)+\epsilon_{i h}\right]+ \\
& G_{i k} \times\left[\beta^{\prime}\left|X_{i}-X_{k}\right|+\delta \log \left(G_{j k}+G_{h k}+1\right)+\epsilon_{i k}\right] .
\end{aligned}
$$

We now provide an overview of the technical use of Assumption 1 in the paper. Assumption 1 a) implies that player $i$ wants to create any link as long as its net return is positive. This allows to represent a PSNE of the network formation game as the solution of a simultaneous discrete choice model. By adding Assumption $1 \mathrm{~b}$ ), one can partition the network formation game into $N$ local games such that the players' payoffs within each local game are not affected by the players' choices outside of it. This is crucial to maintain that the network formation game is in equilibrium if and only if each local game is in equilibrium (if and only if property in Proposition 1). As carefully examined in the next sections, the if and only if property is a powerful result because it helps us substantially mitigate the curse of dimensionality in the analysis. Introducing additional spillover effects into $u_{i}\left(\cdot ; \theta_{u}\right)$ causes the if and only if property to fail and more restrictions are needed to achieve similar simplifications. Lastly, the assumption about the monotonicity of $v_{j}\left(\cdot ; \delta_{j}\right)$ is exploited, together with the if and only property, to show equilibrium existence and to speed the calculation of the integrals which enter the identifying moment inequalities. ${ }^{10}$

Remark 1 concludes by comparing Assumption 1 with restrictions imposed in other papers.

Remark 1. Let us analyse some (de)merits of Assumption 1 through a comparison with the restrictions in Miyauchi (2016), Sheng (2016), de Paula, Richards-Shubik, and Tamer (2018) (hereafter, PST), and Mele (2017).

As we do, Miyauchi and Sheng examine a many-network setting. Unlike here, they focus on reciprocal links, i.e., $G_{i j}=G_{j i} \forall(i, j) \in \mathcal{N}^{2}$. Miyauchi and Sheng's methodologies can handle

\footnotetext{
${ }^{10}$ Note that when $v_{j}\left(\cdot ; \delta_{j}\right)$ is nonlinear, the network formation game may not be a potential game. Potential games can help simplify the econometric analysis, as discussed in Sheng (2016) and Mele (2017).
} 
various spillover effects and are manageable for large $N$. However, the regions of parameters values they characterise are neither proved to be sharp nor studied in relation to the sharp identified set. Moreover, the spillover effects in Miyauchi are required to have a positive sign and the spillover effects in Sheng are assumed positive for some model specifications. Instead, we do not restrict the sign of $\delta$.

PST and Mele examine a one-network setting. PST focus on reciprocal links and allow connections up to distance $D$ to affect utility. However, preferences in PST are such that the players never choose more than $L$ links in total, which, e.g., is not verified in the PHBIs' application of Section $4 .{ }^{11}$ Moreover, according to the PST's framework, the players' observed characteristics are discrete and the players are endowed with a preference shock for each realisation of those characteristics. Thus, potential friends of the same type are viewed as perfect substitutes. Instead, we accommodate richer unobserved heterogeneity by including pair specific preference shocks, which seems more appropriate when dealing with small groups of players. Mele considers nonreciprocal links and includes various spillover effects. However, Mele imposes equilibrium uniqueness by designing a sequential meeting process, while instead we contemplate multiple equilibria.

\subsection{Existence of an equilibrium}

This section proves that the network formation game has at least one equilibrium for every value of the payoff-relevant variables and parameters, by building on results from Berry (1992). Arguments are made of intermediate results which are interesting per se because they highlight aspects of the model playing a key role later in the identification analysis.

We first show that the inequalities in Definition 1 simplify to a system of $N(N-1)$ equations whose solution is an equilibrium of the network formation game.

Lemma 1. (Characterisation of a PSNE of the network formation game) Under Assumption 1 a), $\boldsymbol{G} \in \mathscr{G}$ is a PSNE of the network formation game if and only if

$$
G_{i j}=\mathbb{1}\left\{u_{i j}\left(\boldsymbol{G}_{-\{i \cdot\}}, \boldsymbol{X}, \epsilon ; \theta_{u}\right) \geq 0\right\} \quad \forall(i, j) \in \mathcal{N}^{2} .
$$

We now introduce the local games. For each $j \in \mathcal{N}$, let the section $j$ be the network portion collecting all the nodes of the network and all the links pointing to node $j$. Figures 1 and 2 help clarify the definition of the section $j$.

Let the section $j$ game be the game underlying the formation of the section $j$, i.e., in the section $j$ game the players other than player $j$ simultaneously decide whether to link to $j$. A pure strategy for each player $i \in \mathcal{N} \backslash\{j\}$ is $G_{i j} \in\{0,1\}$ and a pure strategy profile of the game is $G_{. j} \equiv\left(G_{i j} \forall i \in \mathcal{N} \backslash\{j\}\right) \in\{0,1\}^{N-1}$. Under Assumption 1, each player $i \in \mathcal{N} \backslash\{j\}$ gets the payoff $G_{i j} \times\left[z_{i j}(\boldsymbol{X}, \epsilon ; \beta)+v_{j}\left(\sum_{k \in \mathcal{N} \backslash\{i\}} G_{k j} ; \delta\right)\right]$. Agents play PSNE.

\footnotetext{
${ }^{11}$ Indeed, the Italian legal system does not impose limits on the number of board interlocks that a company can form.
} 


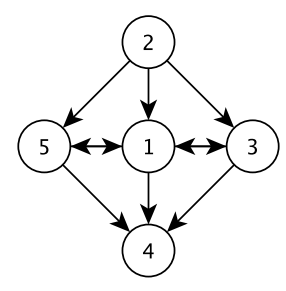

network

Figure 1: Example of network with $N=5$.

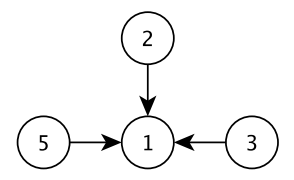

(4)

section 1

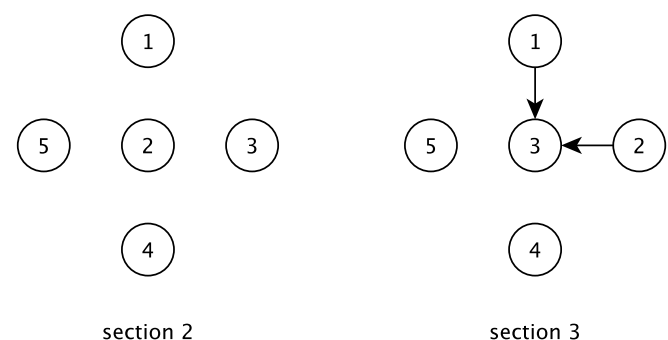

section 2
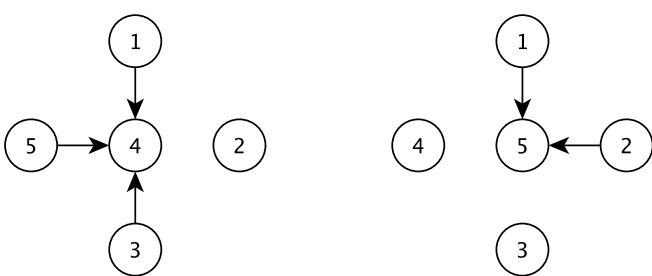

3

section 4

Figure 2: Section 1, section 2, ..., section 5 of the network in Figure 1.

Definition 2. (PSNE of the section $j$ game) Under Assumption 1, $G_{\cdot j} \in\{0,1\}^{N-1}$ is a PSNE of the section $j$ game if

$$
G_{i j}=\mathbb{1}\left\{z_{i j}(\boldsymbol{X}, \epsilon ; \beta)+v_{j}\left(\sum_{k \in \mathcal{N} \backslash\{i\}} G_{k j} ; \delta\right) \geq 0\right\} \quad \forall i \in \mathcal{N} \backslash\{j\}
$$

Note that $\boldsymbol{G} \in \mathscr{G}$ can be written as $(G \cdot 1, \ldots, G \cdot N)$, where $G \cdot 1 \in\{0,1\}^{N-1}, \ldots, G \cdot N \in\{0,1\}^{N-1}$. Moreover, under Assumption 1, the players' payoffs within the section $j$ game depend on $\boldsymbol{G}$ exclusively via $G$.j, i.e., they are not affected by the players' choices outside the section $j$ game. ${ }^{12}$ The if and only if property follows.

Proposition 1. (If and only if property) Under Assumption $1, \boldsymbol{G} \equiv\left(G_{\cdot 1}, G_{\cdot 2}, \ldots, G_{\cdot N}\right) \in \mathscr{G}$ is a PSNE of the network formation game if and only if $G_{\cdot j} \in\{0,1\}^{N-1}$ is a PSNE of the section $j$ game $\forall j \in \mathcal{N}$.

Proposition 1 characterises the set of equilibria of the network formation game as the Cartesian product of the set of equilibria of the section 1 game,..., section $N$ game. Its usefulness goes beyond the existence arguments discussed here. Indeed, Proposition 1 is exploited in Section 3.5 to reduce the number of moment inequalities defining the sharp identified set and to simplify the calculation by simulation of the integrals entering those moment inequalities. ${ }^{13}$

Continuing with the existence proof, it can be noticed that the structure of the section $j$ game when $v_{j}(\cdot ; \delta)$ is monotone decreasing (increasing) is similar to the structure of an entry

\footnotetext{
${ }^{12}$ Nevertheless, up to this point of the exposition, the outcome chosen by the players from the equilibrium set of the section $j$ game can be statistically correlated with the outcome chosen by the players from the equilibrium set of the section $h$ game through the equilibrium selection mechanism.

${ }^{13}$ Proposition 1 holds also under a weaker version of Assumption 1. More details are in Appendix A.
} 
game with substitution (complementary) effects. ${ }^{14}$ Existence of a PSNE of the section $j$ game when $v_{j}(\cdot ; \delta)$ is monotone increasing is guaranteed by Tarski's fixed point theorem. Existence of a PSNE of the section $j$ game when $v_{j}(\cdot ; \delta)$ is monotone decreasing can be shown by adapting to our model the constructive proof developed by Berry (1992) to prove equilibrium existence in an entry game with substitution effects. Merging these results, the section $j$ game has an equilibrium $\forall j \in \mathcal{N}$.

Proposition 2. (Existence of a PSNE of the section $j$ game) Under Assumption 1, there exists a PSNE of the section $j$ game $\forall j \in \mathcal{N}$.

The way in which existence of a local equilibrium is verified helps us determine some features of the set of equilibria of the section $j$ game, which are used in Section 3.5 to simplify further the calculation of the integrals entering the identifying moment inequalities. In particular, when $v_{j}(\cdot ; \delta)$ is monotone increasing, Tarski's fixed point theorem ensures existence of the greatest and least equilibria of the section $j$ game. When $v_{j}(\cdot ; \delta)$ is monotone decreasing, one can mimic another result from Berry (1992) stating that in an entry game with substitution effects all the equilibria are characterised by the same number of firms entering the market.

Corollary 1. (Characterising the set of PSNE of the section $j$ game) Under Assumption 1, when $v_{j}(\cdot ; \delta)$ is monotone increasing, there exists the greatest and least PSNE of the section $j$ game, $\forall j \in \mathcal{N}$. Under Assumption 1 , when $v_{j}(\cdot ; \delta)$ is monotone decreasing, all the PSNE of the section $j$ game are characterised by the same number, $n_{j}^{*} \in\{0,1, \ldots, N-1\}$, of players connecting to player $j, \forall j \in \mathcal{N}$.

Continuing with the existence proof, by combining Propositions 1 and 2, it can be claimed that the network formation game has at least one equilibrium.

Corollary 2. (Existence of a PSNE of the network formation game) Under Assumption 1, there exists a PSNE of the network formation game.

Remark 2 concludes by comparing section $1, \ldots$, section $N$ with other local network decompositions proposed in the literature.

Remark 2. Also Sheng (2016) and PST suggest to decompose networks into local parts to mitigate the various challenges hampering estimation. However, they use local network decompositions different from our.

Sheng focuses on subnetworks. More precisely, take any subset of nodes, $\mathscr{A} \subseteq \mathcal{N}$. The subnetwork $\mathscr{A}$ is the submatrix of $\boldsymbol{G}$ containing all the links connecting the nodes in $\mathscr{A}$. For example, consider the network in Figure 1 and let $\mathscr{A} \equiv\{1,4,5\}$. Then, the subnetwork $\mathscr{A}$ is graphically represented in Figure 3. Let the subnetwork $\mathscr{A}$ game be the game underlying the formation of the subnetwork $\mathscr{A}$. Sheng characterises an identified set by constructing moment inequalities based on the equilibria of subnetwork games, for various subnetworks. On one hand, such a strategy implies that the number of moment inequalities does not depend anymore on $N$, but it depends only on the subnetworks' sizes. Therefore, by selecting sufficiently small subnetworks, the number of moment inequalities is kept limited. On the other hand, the players'

\footnotetext{
${ }^{14}$ Just replace player $j$ with the entry market.
} 


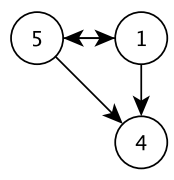

subnetwork $\{1,4,5\}$

Figure 3: Subnetwork $\{1,4,5\}$ of the network in Figure 1.

strategy profiles are overlapping across subnetwork games. Thus, collections of subnetwork games do not satisfy the if and only if property. The failure of the if and only if property has two consequences. First, the characterised identified set is usually non-sharp and it is difficult to provide sufficient conditions under which it is sharp. Second, the characterised identified set typically involves moment inequalities containing integrals whose computation by simulation still requires getting the set of equilibria of the entire game. This is because a local equilibrium is acceptable only when compatible with a global equilibrium. As getting the set of equilibria of the entire game can be computationally expensive, further assumptions (e.g., sign restrictions) are proposed by Sheng in some scenarios to facilitate the analysis.

PST focus on network types. More precisely, take any player $i \in \mathcal{N}$. Player $i$ 's network type is the submatrix of $\boldsymbol{G}$ which contains all the links affecting $i$ 's payoff. For example, consider the network in Figure 1. Under Assumption 1, player 5's network type is graphically represented in Figure 4.

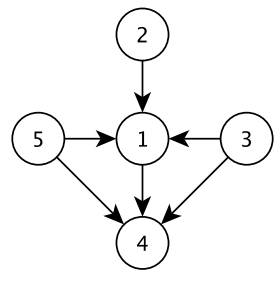

player 5 ' network type

Figure 4: Player 5's network type of the network in Figure 1 under Assumption 1.

There are two main differences between our local network decomposition and the one in PST. First, similarly to what discussed above about Sheng, collections of games underlying the formation of network types do not satisfy the if and only if property. Second, our local network decomposition serves to simplify the moment inequalities arising in a many-network setting. Instead, PST use network types to construct a law of large numbers in a one-network setting. $\diamond$

\subsection{Assumptions on the data generating process}

This section describes the data generating process (hereafter, DGP). From now on, capital letters denote random variables and small case letters are used for their realisations.

Assumption 2. (DGP)

a) An integer $N$ is drawn from $\mathbb{N} \backslash\{1,2\}$. $N$ agents, endowed with characteristics collected in $(\boldsymbol{X}, \epsilon)$, are drawn from the population and play the network formation game described in 
Section 2 with payoffs satisfying Assumption 1. An equilibrium network, $\boldsymbol{G}$, arises. If the set of equilibria is non-singleton, then the players coordinate on an outcome from the equilibrium set according to a selection mechanism that is unobserved by the researcher. ${ }^{15}$

The procedure is repeated $M$ times, with $M$ large. A sample of observations, $\left\{n_{m}, \boldsymbol{x}_{m}, \boldsymbol{g}_{m}\right\}_{m=1}^{M}$, is collected. The sampling scheme is designed such that the population probability distribution of $\boldsymbol{G}$ conditional on $(N, \boldsymbol{X})$ (hereafter, empirical probability distribution of the observables) is nonparametrically identified. ${ }^{16,17}$

b) $\epsilon$ is continuously distributed on $\mathbb{R}^{N(N-1)}$, independently of $(N, \boldsymbol{X})$, with cdf denoted by $F\left(\cdot ; \theta_{\epsilon}\right)$, where $\theta_{\epsilon} \in \Theta_{\epsilon} \subset \mathbb{R}^{d_{\epsilon}}$ is a vector of parameters. ${ }^{18}$

c) $\Theta \equiv\left(\Theta_{u} \cup \Theta_{\epsilon}\right) \ni \theta \equiv\left(\theta_{u}, \theta_{\epsilon}\right)$ is compact.

d) All the random variables, vectors, and matrices are defined on the same probability space, $(\Omega, \mathscr{F}, \mathbb{P})$.

Assumption 2 is standard in models featuring many-network asymptotics. In what follows, we provide some comments about the role of the players' labels. In particular, Assumption 2 allows for three alternative scenarios. In the first scenario, the players' identities are the same across the observed networks. In this case, the labels assigned to the players correspond to their identities, i.e., player $i$ in network $m$ is the same as player $i$ in network $m^{\prime}$.

In the second scenario, the players' identities vary across the observed networks. However, the observed networks have the same size and the players have fixed roles. In this case, the labels assigned to the players correspond to their roles, i.e., player $i$ in network $m$ has the same role as player $i$ in network $m^{\prime}$. For example, suppose that the players are firms and that the researcher can rank them unambiguously with respect to size within each network. Then, the probability of observing a network containing a link from firm $i$ to firm $j$ is interpreted as the probability of observing a network containing a link from the $i$-th biggest firm to the $j$-th biggest firm.

In the third scenario, the players' identities vary across the observed networks and the players have no roles. In this case, the labels are assigned arbitrarily to the players within each network. For example, suppose that the players are firms and that the only feature observed by the researcher is their profitability in brackets. Within each network, it is likely that there are two or more firms featuring the same profitability in brackets. Therefore, the researcher cannot unambiguously rank the firms within each network. Such arbitrariness of labelling does not affect

\footnotetext{
${ }^{15}$ Note that Section 3.2 shows that the set of equilibria is always non-empty.

${ }^{16}$ "All that is needed is for the law of large numbers to hold." (Ciliberto and Tamer, 2009, p.1799), e.g., an i.i.d. sampling scheme or a stationary and ergodic sampling scheme.

${ }^{17}$ Note that the number of players, $n_{m}$, is allowed to vary across $m=1, \ldots, M$.

${ }^{18}$ Note that all the results in Section 3 are valid also if we assume that $\epsilon$ is continuously distributed on $\mathbb{R}^{N(N-1)}$ conditional on $(N, \boldsymbol{X})$, with conditional cdf denoted by $F\left(\cdot \mid N, \boldsymbol{X} ; \theta_{\epsilon}\right)$ and known up to $\theta_{\epsilon}$. Moreover, in principle, one could carry on the identification analysis even without assuming that the (conditional) cdf of $\epsilon$ belongs to a parametric family. However, in practice, constructing the resulting sharp identified set would be an intractable exercise because it requires searching for an infinite dimensional function, i.e., an admissible (conditional) cdf of $\epsilon$, for each value of the payoff parameters. Some recent studies investigate this issue in the absence of multiple equilibria (Torgovitsky, 2018) or in the presence of multiple equilibria (Chesher and Rosen, 2019). We believe that extending similar results to our framework deserves a separate research project.
} 
identification, i.e., the identification arguments developed in the next sections hold for any label assignment. However, when doing inference and replacing probabilities with sample analogues, the researcher needs to ensure that the chosen label assignment does not affect estimates. One way to achieve that is to add sufficient conditions such that the probability distribution of $\boldsymbol{G}$ conditional on $(N, \boldsymbol{X})$ is invariant under permutations of the labels, as suggested by Sheng (2016). This amounts to impose joint exchangeability of networks (Kallenberg, 2005). ${ }^{19}$ The empirical illustration in Section 4 falls in the third scenario and we rely on joint exchangeability of networks when conducting inference, as explained in Appendix ?? of the supplementary material.

\subsection{From multiple equilibria to partial identification}

The spillover effect described in Assumption 1 implies that the network formation game can have multiple equilibria. This means that the values of the exogenous variables do not uniquely pin down the values of the endogenous variables. Equivalently, there is no unique mapping from the parameters and the exogenous variables to the endogenous variables.

As the equilibrium selection mechanism is unobserved by the researcher and economic theory provides no clear guidance regarding its form, the econometric analysis proceeds by allowing for multiple equilibria. Under multiple equilibria, deriving sufficient conditions for point identification of the parameters is difficult. ${ }^{20}$ Therefore, the study discusses partial identification of the parameters, i.e., it admits the possibility that there may be more than one parameter value able to generate the empirical probability distribution of the observables under the model's assumptions.

\subsection{The sharp identified set}

The sharp identified set, $\Theta^{\star}$, is the set of parameter values for which one can find an equilibrium selection mechanism that delivers the empirical joint probability distribution of the observables when combined with the model's assumptions. The definition of $\Theta^{\star}$ does not facilitate inference because it involves the equilibrium selection mechanism which is an infinite dimensional function. This section provides a characterisation of $\Theta^{\star}$ that makes inference on it computationally workable for sufficiently small networks, by building on results from Beresteanu, Molchanov, and Molinari (2011) (hereafter, BMM).

More precisely, BMM show that the sharp identified set in the class of models with convex moment predictions can be expressed as the set of parameter values satisfying a finite collection of inequalities. Such inequalities are obtained by bounding the empirical probability distribution of the observables and do not contain the equilibrium selection mechanism. Under Assumptions 1 and 2, this model belongs to the class of models analysed by BMM. Therefore, we start our discussion by representing $\Theta^{\star}$ according to BMM's approach.

\footnotetext{
${ }^{19}$ More formally, joint exchangeability of networks is defined as: $\mathbb{P}(\boldsymbol{G} \in K \mid N=n, \boldsymbol{X}=\boldsymbol{x})=\mathbb{P}\left(\boldsymbol{G} \in K^{\varphi} \mid N=\right.$ $n, \boldsymbol{X}=\boldsymbol{x}^{\varphi}$ ), for every permutation $\varphi$ of the labels in $\mathcal{N}, \forall K \subseteq \mathscr{G}, \forall \boldsymbol{x} \in \mathscr{X}^{n}$, and $\forall n \in \mathcal{N}$, where $K^{\varphi}$ and $\boldsymbol{x}^{\varphi}$ are obtained by applying $\varphi$ respectively to $K$ and $\boldsymbol{x}$.

${ }^{20}$ Conversely, note that fixing the equilibrium selection mechanism does not ensure point identification of the parameters.
} 
For simplicity of exposition and without loss of generality, let us assume that all the observed networks have the same size, i.e., $N$ is a degenerate random variable with support $\{n\}$, for any $n \in \mathbb{N} \backslash\{1,2\}$. Let $\mathscr{A}_{\mathscr{G}} \subset \mathscr{K}_{\mathscr{G}}$ be the collection of non-empty finite subsets of $\mathscr{G}$ obtained by taking the Cartesian product of all the possible ordered $n$-tuples with repetition from $\mathscr{K}_{\{0,1\}^{n-1} \cdot{ }^{21}}$ For each $\theta \in \Theta$, consider the random closed set of equilibria of the network formation game, $\delta_{\theta_{u}}(\boldsymbol{X}, \epsilon): \Omega \rightarrow A_{g}$. Note that $\delta_{\theta_{u}}(\boldsymbol{X}, \epsilon)$ takes values in $\mathcal{A}_{\boldsymbol{G}}$ by the if and only if property. Under Assumptions 1 and 2, Theorem D.2 in BMM suggests that

$$
\Theta^{\star}=\left\{\theta \in \Theta \mid \mathbb{P}(\boldsymbol{G} \in K \mid \boldsymbol{X}=\boldsymbol{x}) \leq T_{\boldsymbol{x}, \theta}(K) \forall K \in \mathscr{K}_{\mathscr{G}}, \forall \boldsymbol{x} \in \mathscr{X}^{n} \text { a.s. }\right\},
$$

where $T_{\boldsymbol{x}, \theta}: \mathscr{K}_{\varphi} \rightarrow[0,1]$ is the capacity functional of $\delta_{\theta_{u}}(\boldsymbol{X}, \epsilon)$ conditional on $\boldsymbol{X}=\boldsymbol{x}$, prescribed by

$$
T_{\boldsymbol{x}, \theta}(K) \equiv \mathbb{P}\left(\delta_{\theta_{u}}(\boldsymbol{X}, \epsilon) \cap K \neq \emptyset \mid \boldsymbol{X}=\boldsymbol{x} ; \theta\right)=\int_{e \in \mathbb{R}^{n(n-1)} \text { s.t. } \delta_{\theta_{u}}(\boldsymbol{x}, e) \cap K \neq \emptyset} d F\left(e ; \theta_{\epsilon}\right),
$$

$\forall K \in \mathscr{K}_{\mathscr{g}} \cdot{ }^{22,23}$ Each inequality in (5) is known as Artstein's inequality, for a total of $2^{2^{n(n-1)}}-2$ Artstein's inequalities $\forall \theta \in \Theta$ and $\forall \boldsymbol{x} \in \mathscr{X}^{n}$.

Unfortunately, conducting inference on $\Theta^{\star}$ as characterised in (5) is computationally unmanageable even for $n=3$ because it requires checking the violation in the data of an overwhelming amount of inequalities. ${ }^{24}$ Moreover, the capacity functionals entering the inequalities in (5) depend on the equilibria of the whole network formation game. Hence, they can be burdensome to calculate by simulation if one naively opts for enumerating all the possible $2^{n(n-1)}$ networks and verifying whether each candidate satisfies the equilibrium conditions for each drawn realisation of the taste shocks.

As an alternative route, in what follows we consider the region of parameter values cropped by bounding the empirical probability distributions of the outcomes of the section 1 game, ..., section $n$ game, rather than of the entire network formation game. We argue that, under some restrictions on preference shocks and equilibrium selection, such an approach mitigates the concerns illustrated above and makes inference on $\Theta^{\star}$ computationally workable when networks are sufficiently small.

More formally, for each $\theta \in \Theta$ and $j \in \mathcal{N}$, let $\mathcal{S}_{j, \theta_{u}}\left(\boldsymbol{X}, \epsilon_{\cdot j}\right): \Omega \rightarrow \mathscr{K}_{\{0,1\}^{n-1}}$ be the random closed set of equilibria of the section $j$ game, where $\epsilon_{. j} \equiv\left(\epsilon_{i j} \forall i \in \mathcal{N} \backslash\{j\}\right) .{ }^{25}$ An additional assumption is now introduced.

Assumption 3. (Independence)

a) The random vectors in the sequence $\left\{\epsilon_{\cdot j}\right\}_{j \in \mathcal{N}}$ are mutually independent.

b) The section 1 game, ..., section $n$ game are endowed with mutually independent equilibrium

\footnotetext{
${ }^{21}\left|\mathscr{A}_{\mathscr{G}}\right|=\left(2^{2^{n-1}}-1\right)^{n}<\left|\mathscr{K}_{\mathscr{G}}\right|=2^{2^{n(n-1)}}-1$. See Appendix D for more details on how $\mathscr{A}_{\mathscr{G}}$ is constructed.

${ }^{22}$ The same characterisation can be obtained by applying some results from Galichon and Henry $(2006 ; 2011)$.

${ }^{23}$ Throughout the paper and with some abuse of notation, "; $\theta$ " inside a probability indicates that the value of the considered probability, as predicted by the model, depends on $\theta$.

${ }^{24}$ For $n=3,2^{2^{n(n-1)}}-2 \approx 1.845 \times 10^{19}$. For $n \geq 4,2^{2^{n(n-1)}}-2>10^{80}$

${ }^{25}$ Note that $\epsilon \equiv(\epsilon \cdot 1, \ldots, \epsilon \cdot n)$.
} 
selection mechanisms, i.e.,

$$
\begin{aligned}
& \mathbb{P}\left(G_{\cdot 1}=g_{\cdot 1}, \ldots, G_{\cdot n}=g_{\cdot n} \mid \boldsymbol{X}, \epsilon\right)=\Pi_{j=1}^{n} \mathbb{P}\left(G_{\cdot j}=g_{\cdot j} \mid \boldsymbol{X}, \epsilon_{\cdot j}\right), \\
& \forall \boldsymbol{g} \equiv\left(g_{\cdot 1}, \ldots, g_{\cdot n}\right) \in \mathscr{G} \text { and } \forall \boldsymbol{x} \in \mathscr{X}^{n} .
\end{aligned}
$$

Assumption 3 a) restricts Assumption 2 b) by not allowing $\epsilon_{\cdot j}$ to be correlated with $\epsilon_{\cdot h}$, $\forall(j, h) \in \mathcal{N}^{2}$. In particular, under Assumption 3 a), the agents' taste shocks can be correlated through link target-specific unobserved heterogeneity, but not through link source-specific unobserved heterogeneity. For example, Assumption 3 a) is satisfied if $\epsilon_{i j} \equiv \alpha_{j}+\xi_{i j} \forall(i, j) \in \mathcal{N}^{2}$. Assumption 3 a) is violated if $\epsilon_{i j} \equiv \alpha_{i}+\beta_{j}+\xi_{i j}$ for some $(i, j) \in \mathcal{N}^{2}$. Other papers on network formation impose $\left\{\epsilon_{i j}\right\}_{\forall(i, j) \in \mathcal{N}^{2}}$ i.i.d., which is stronger than Assumption 3 a) (e.g., Menzel, 2016; Sheng, 2016). Additionally, note that Assumption 3 a) allows for network sparsity by letting the correlation among the preference shocks within $\epsilon_{\cdot j}$ to appropriately depend on $n \forall j \in \mathcal{N}$, so that nodes have a non-negligible probability of remaining isolated as $n$ grows. More details on this are in Leung (2015).

Assumption $3 \mathrm{~b}$ ) is a local coordination condition. It strengthens the if and only if property by requiring that the players of each local game coordinate on the selection of an equilibrium independently across the local games. In other words, Assumption $3 \mathrm{~b}$ ) excludes that the players of the section $j$ game select an outcome from the set of equilibria of the section $j$ game by looking at the equilibrium selection rule adopted by the players of the section $h$ game, $\forall(j, h) \in \mathcal{N}^{2}$. For example, Assumption $3 \mathrm{~b}$ ) is satisfied if the players of the section $j$ game roll a die to select an outcome from the set of equilibria of the section $j$ game, independently across $j$. Assumption $3 \mathrm{~b}$ ) is met if the players of the section $j$ game roll a die to select the player who should be privileged by the local bargaining process, independently across $j$. Myopic best-response dynamics are contemplated by Assumption $3 \mathrm{~b}$ ), i.e., when the players of the section $j$ game meet sequentially at random and choose which links to form by maximizing current utilities, $\forall j \in \mathcal{N}$. Many papers in the literature assume equilibrium selection via myopic best-response dynamics (e.g., Christakis, et al., 2010; Mele, 2017). Assumption 3 b) is violated if all the local games systematically coordinate on an equilibrium penalising player $k$. A requirement similar to Assumption $3 \mathrm{~b}$ ) is imposed by Leung (2019) in a one-network setting and is referred to as "decentralised selection".

Lastly, some implications of Assumption 3 can be tested. In particular, under Assumptions 1,2 , and 3 , it holds that

$$
\mathbb{P}(\boldsymbol{G}=\boldsymbol{g} \mid \boldsymbol{X})=\Pi_{j=1}^{n} \mathbb{P}\left(G_{\cdot j}=g_{\cdot j} \mid \boldsymbol{X}\right) \quad \forall \boldsymbol{g} \equiv\left(g_{\cdot 1}, \ldots, g_{\cdot n}\right) \in \mathscr{G},
$$

i.e., the random vectors in the sequence $\left\{G_{. j}\right\}_{j \in \mathcal{N}}$ are mutually independent conditional on $\boldsymbol{X} .{ }^{26}$ This null hypothesis can be verified using any method in the literature for testing conditional independence, e.g., Fukumizu, et al. (2008) and Zhang, et al. (2011).

\footnotetext{
${ }^{26}$ This is formally shown in step 2.3 of the proof of Proposition 3 in Appendix C.
} 
Continuing with identification, define the set

$$
\begin{aligned}
\Theta^{\star \star} \equiv\left\{\theta \in \Theta \mid \mathbb{P}\left(G_{\cdot j} \in K_{j} \mid \boldsymbol{X}=\boldsymbol{x}\right) \leq\right. & T_{j, \boldsymbol{x}, \theta}\left(K_{j}\right) \\
& \left.\forall K_{j} \in \mathscr{K}_{\{0,1\}^{n-1}}, \forall j \in \mathcal{N}, \forall \boldsymbol{x} \in \mathscr{X}^{n} \text { a.s. }\right\},
\end{aligned}
$$

where $T_{j, \boldsymbol{x}, \theta}: \mathscr{K}_{\{0,1\}^{n-1}} \rightarrow[0,1]$ is the capacity functional of $\mathcal{\delta}_{j, \theta_{u}}\left(\boldsymbol{X}, \epsilon_{\cdot j}\right)$ conditional on $\boldsymbol{X}=\boldsymbol{x}$, prescribed by

$$
\begin{aligned}
T_{j, \boldsymbol{x}, \theta}\left(K_{j}\right) \equiv \mathbb{P}\left(\mathcal{S}_{j, \theta_{u}}\left(\boldsymbol{X}, \epsilon_{. j}\right) \cap K_{j} \neq \emptyset \mid \boldsymbol{X}=\boldsymbol{x} ; \theta\right) \\
=\int_{e_{. j} \in \mathbb{R}^{n-1} \text { s.t. } \delta_{j, \theta_{u}}\left(\boldsymbol{x}, e_{. j}\right) \cap K \neq \emptyset} d F_{j}\left(e_{. j} ; \theta_{\epsilon}\right),
\end{aligned}
$$

$\forall K_{j} \in \mathscr{K}_{\{0,1\}^{n-1}}$, where $F_{j}\left(\cdot ; \theta_{\epsilon}\right)$ denotes $\epsilon \cdot j^{\prime}$ 's cdf.

Proposition 3. (Sharp identified set under Assumptions 1, 2, 3) (i) Under Assumptions 1 and $2, \Theta^{\star \star} \supseteq \Theta^{\star}$. (ii) Under Assumptions 1,2 , and $3, \Theta^{\star \star}=\Theta^{\star}$.

Intuitively, Proposition 3 can be read as follows. If the researcher is not willing to impose Assumption 3, then $\Theta^{\star \star}$ is an outer set of the sharp identified set. This is because, by ignoring the relationships among the local games, $\Theta^{\star \star}$ does not fully explore the empirical content of the model. When Assumption 3 is included, the relationships among the local games are voided of their empirical content. Therefore, considering the information provided by the probability distributions of the local game outcomes is sufficient for sharpness. The proof of Proposition 3 in Appendix C formalises these arguments.

From a technical point of view, Proposition 3 shows that "thinking locally" entails a significant reduction in the number of inequalities needed for sharpness. This is because the bounded probability distributions have fewer mass points. In particular, the number of inequalities is shortened to $n\left(2^{2^{n-1}}-2\right)<<2^{2^{n(n-1)}}-2$, with a reducing factor of order $O\left(2^{2^{n^{2}}} / n\right) .{ }^{27}$

Furthermore, differently from (5), the capacity functionals entering Artstein's inequalities in (6) are easily calculable via the simple frequency simulator proposed by McFadden (1989) and Pakes and Pollard (1989), for two reasons. First, when bounding the empirical probability distribution of $G_{. j}$, the if and only if property implies that the involved capacity functionals depend only on the set of equilibria of the section $j$ game. Consequently, during the simulation procedure, the researcher can look separately for the equilibria of the section 1 game, ..., section $n$ game, without needing to verify whether a potential equilibrium of the section $j$ game is compatible with an equilibrium of the whole network formation game $\forall j \in \mathcal{N}$. Second, in order to determine the equilibria of the section $j$ game, the researcher does not have to check the equilibrium conditions for each possible realisation of $G_{\cdot j}$. Instead, the researcher can exploit Corollary 1 to refine ex-ante the collection of realisations of $G_{\cdot j}$ which can strive for being equilibria. Specifically, if a candidate parameter value is such that $v_{j}(\cdot ; \delta)$ is monotone increasing, Tarski's fixed point theorem guarantees existence of the greatest and least equilibria of the section $j$ game. These two equilibria can be computed from the best-response dynamics, where the number of iterations for convergence is no more than $(n-1)^{2}$ (Topkis 1979). It follows that the

\footnotetext{
${ }^{27}$ For $n=3, n\left(2^{2^{n-1}}-2\right)=42$. For $n=4, n\left(2^{2^{n-1}}-2\right)=1016$. For $n=5, n\left(2^{2^{n-1}}-2\right)=327670$.
} 
researcher only has to verify whether the realisations of $G \cdot j$ lying between the greatest and least equilibria are equilibria of the section $j$ game. If a candidate parameter value is such that $v_{j}(\cdot ; \delta)$ is monotone decreasing, all the equilibria of the section $j$ game are characterised by the same number, $n_{j}^{*}$, of players linking to player $j . n_{j}^{*}$ can be obtained by implementing the constructive algorithm used to show existence of an equilibrium of the section $j$ game. ${ }^{28}$ Thus, the researcher only has to check whether the realisations of $G_{. j}$ characterised by $n_{j}^{*}$ players linking to player $j$ are equilibria of the section $j$ game. The number of realisations of $G_{. j}$ characterised by $n_{j}^{*}$ players linking to player $j$ is $\left(\begin{array}{c}n-1 \\ n_{j}^{*}\end{array}\right)$ and $\max _{n_{j}^{*} \in\{0, \ldots, n-1\}}\left(\begin{array}{c}n-1 \\ n_{j}^{*}\end{array}\right)$ is $o\left(2^{n-1}\right)$.

Before concluding, we recognise that the curse of dimensionality in the amount of inequalities to consider for sharpness persists, despite substantially attenuated by the local approach. This is an inherent aspect of sharpness in games with multiple equilibria which cannot be eliminated, but just weakened, without further assumptions. When networks have sizes that make inference on (6) unworkable, we recommend using a sub-collection of inequalities. As suggested by Ciliberto and Tamer (2009) for entry games, one possibility is to consider the outer set of parameter values such that the empirical probability of each realisation of $G_{. j}$ is between the probability of such a realisation being the unique equilibrium of the section $j$ game, and the probability of such a realisation being a possible equilibrium of the section $j$ game, $\forall j \in \mathcal{N}$. That is,

$$
\begin{aligned}
\Theta^{\mathrm{o}} \equiv\{ & \left\{\theta \in \Theta \mid \mathbb{P}\left(G_{\cdot j} \in\{0,1\}^{n-1} \backslash\left\{g_{\cdot j}\right\} \mid \boldsymbol{X}=\boldsymbol{x}\right) \leq T_{j, \boldsymbol{x}, \theta}\left(\{0,1\}^{n-1} \backslash\left\{g_{\cdot j}\right\}\right)\right. \\
& \left.\mathbb{P}\left(G_{\cdot j}=g_{\cdot j} \mid \boldsymbol{X}=\boldsymbol{x}\right) \leq T_{j, \boldsymbol{x}, \theta}\left(\left\{g_{\cdot j}\right\}\right) \forall g_{\cdot j} \in\{0,1\}^{n-1}, \forall j \in \mathcal{N}, \forall \boldsymbol{x} \in X^{n} \text { a.s. }\right\} .
\end{aligned}
$$

Note that, under Assumptions 1 and $2, \Theta^{\circ} \supseteq \Theta^{\star}$ because $\Theta^{\circ} \supseteq \Theta^{\star \star}$ by construction and $\Theta^{\star \star} \supseteq \Theta^{\star}$ by Proposition 3 (i). If Assumption 3 is additionally satisfied, we expect $\Theta^{\circ}$ to become sharper.

We conclude by investigating the shapes of $\Theta^{\star}$ and $\Theta^{\circ}$ through some simulations. To generate the data, we assume

$$
u_{i}\left(\boldsymbol{G}, \boldsymbol{X}, \epsilon ; \theta_{u}\right) \equiv \sum_{j=1}^{N} G_{i j} \times\left[\beta \times\left|X_{i}-X_{j}\right|+\delta \times \sum_{k \neq i}^{N} G_{k j}+\epsilon_{i j}\right],
$$

with $(\beta, \delta)=(-0.5,0.4)$ (hereafter, complementary effect case) or $(\beta, \delta)=(0.8,-0.9)$ (hereafter, substitution effect case), $\left\{X_{i}\right\}_{\forall i \in \mathcal{N}}$ i.i.d., $X_{i} \sim \operatorname{Unif}(\{0,1\}),\left\{\epsilon_{i j}\right\}_{\forall(i, j) \in \mathcal{N}^{2}}$ i.i.d. and independent of $\boldsymbol{X}$, and $\epsilon_{i j} \sim \mathcal{N}(0,1)$. In case of multiple equilibria, we let players select one outcome uniformly at random from the equilibrium set of each local game, independently across local games. Hence, Assumption 3 is satisfied and $\Theta^{\star}=\Theta^{\star \star}$. Figure 5 plots $\Theta^{\star}$ and $\Theta^{\circ}$ for $N=3,4,5$. The three panels on the left are for the complementary effect case. The three panels on the right are for the substitution effect case. In all the designs but one $(N=4$, complementary effect case), the loss of information induced by $\Theta^{\circ}$ with respect to $\Theta^{\star}$ is indiscernible. Figure 6 plots $\Theta^{\circ}$ and the true parameter vector for $N=10,12,14$. In all the designs, $\Theta^{\circ}$ provides extremely informative bounds for the true parameter vector. Interestingly, in the complementary effect case $\Theta^{\circ}$ seems to become larger as $N$ grows, while the opposite trend takes place in the substitution effect case. $^{29}$

\footnotetext{
${ }^{28}$ See the proof of Proposition 2 in Appendix C.

${ }^{29}$ The identified sets in Figures 5 and 6 seem convex. Convexity is not a generic feature of the model.
} 

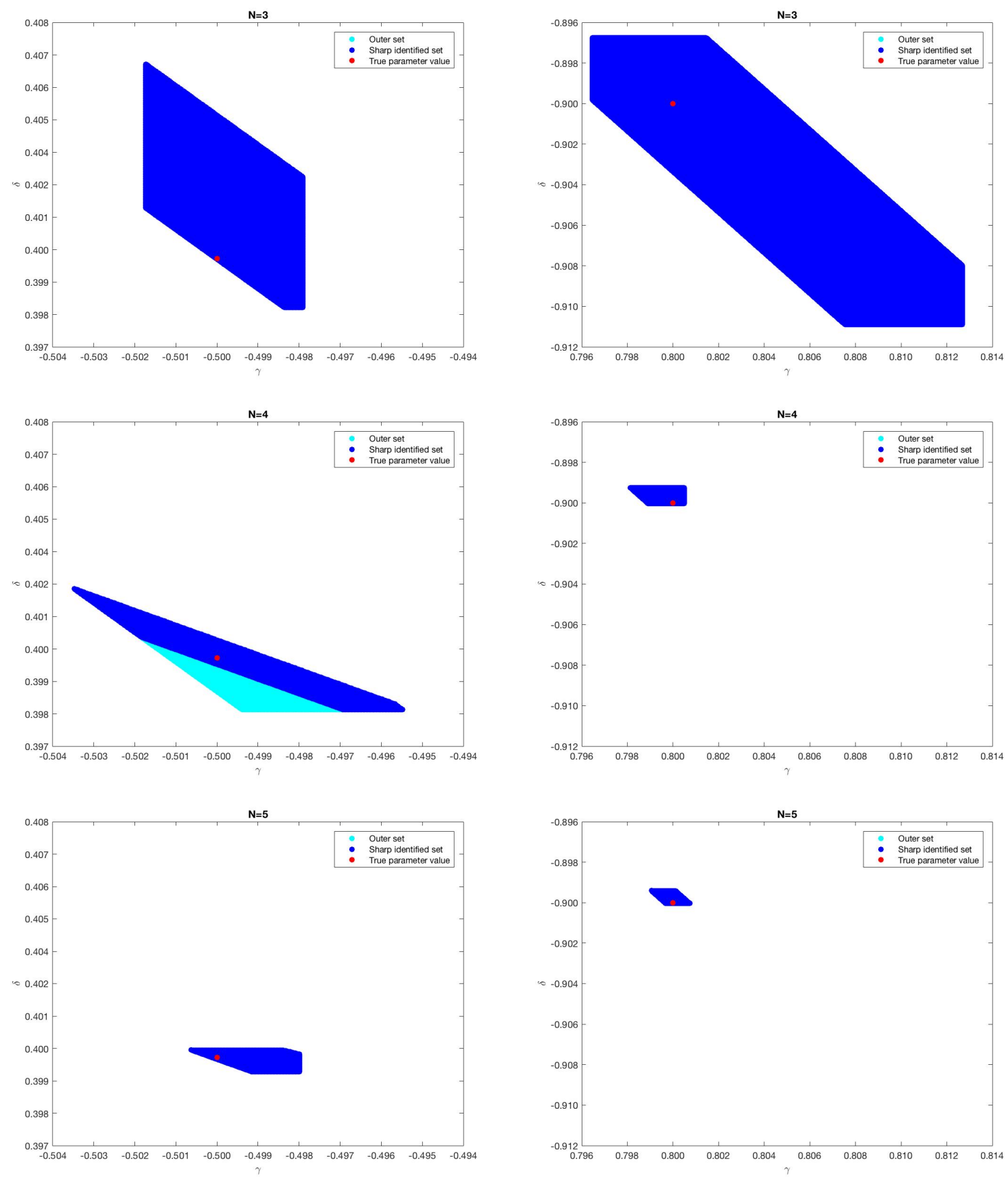

Figure 5: $\Theta^{\star}, \Theta^{\circ}$, and the true parameter vector are plotted for $N=3,4,5$. The three panels on the left are obtained from specification $(8)$ with $(\beta, \delta)=(-0.5,0.4)$. The three panels on the right are obtained from specification $(8)$ with $(\beta, \delta)=(0.8,-0.9)$. 

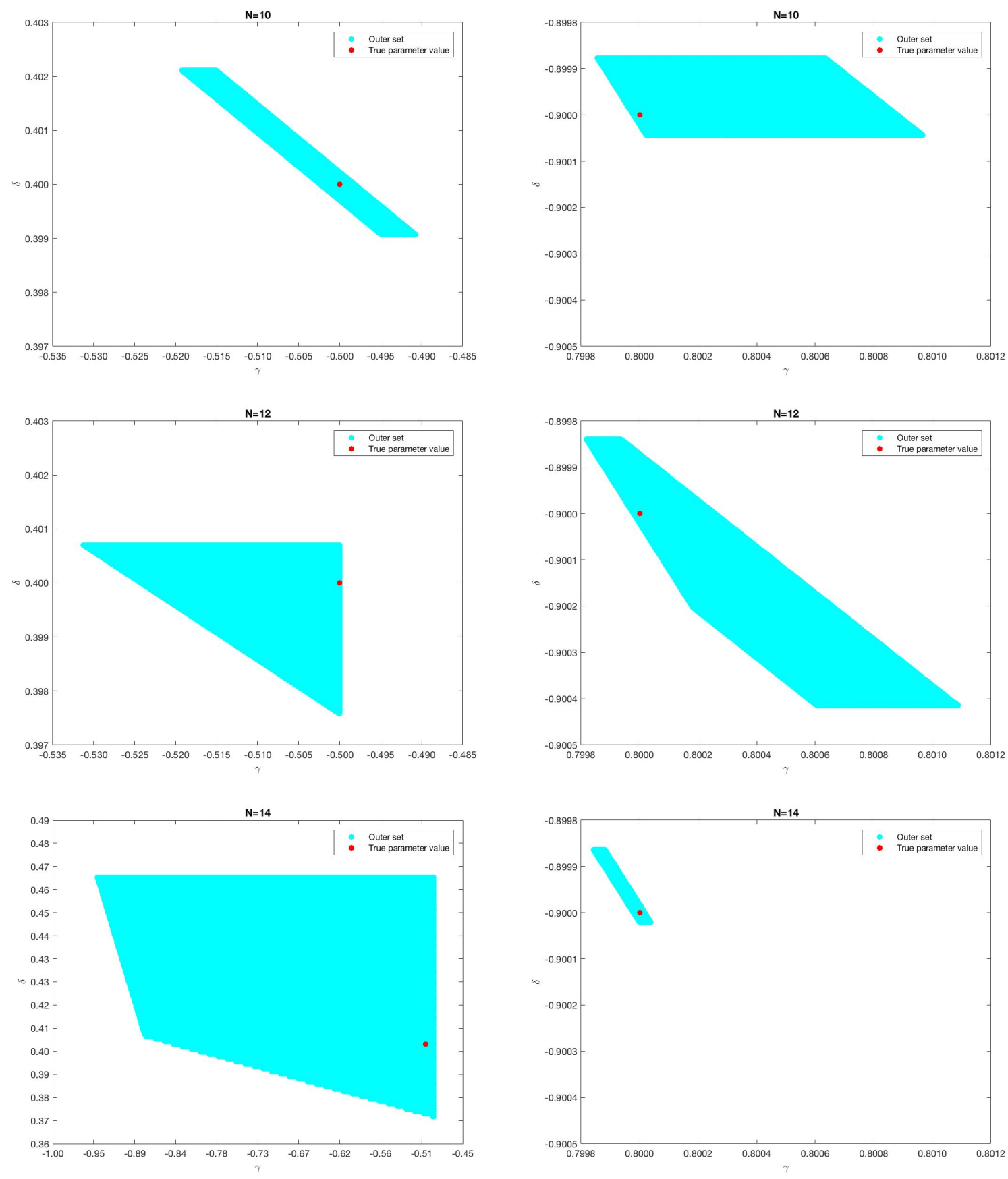

Figure 6: $\Theta^{\circ}$ and the true parameter vector are plotted for $N=10,12,14$. The three panels on the left are obtained from specification $(8)$ with $(\beta, \delta)=(-0.5,0.4)$. The three panels on the right are obtained from specification $(8)$ with $(\beta, \delta)=(0.8,-0.9)$. 
Looking closer at our simulations, we found that the set of equilibria of the local games vary little across convex combinations of parameters in the identified sets. This, combined with limitations in numerical precision, may be the cause of the (apparently) convex shapes. 


\section{Empirical illustration}

This section applies our methodology to the study of Italian firms' incentives for creating PHBIs. Before proceeding, note that, for simplicity of exposition and without loss of generality, the identification analysis of Section 3 focuses on the unilateral case. Appendix B explains how all the results can be replicated for the bilateral case. The formation of PHBIs is an example of bilateral agreement process.

\subsection{The Italian context}

The firms considered in the empirical illustration are Italian joint stock companies (Societá per Azioni). Joint stock companies are business entities where the shareholders' liability is limited to the nominal value of held shares. They represent the largest organisations in Italy. They are not necessarily listed on the stock exchange and are governed by a board of directors. The board of directors is a collegial body appointed by the shareholders who are free to choose its size. The board of directors can delegate its executive duties to one or more of its members (Amministratori Delegati). If the mandate is conferred, then the executives have to report to the board with a frequency determined by the company's statute and, in any case, at least every six months. The non-executives can ask the executives to provide the board with any information related to the management of the firm and are supposed to monitor and inspire the executives' conduct for the good of the company (Articles 2381, 2392, and 2393 of the Italian Civil Code).

The sharing of board members between joint stock companies has been a distinguishing feature of Italian capitalism since the end of the nineteenth century (Luzzatto Fegiz, 1928; Bianco and Pagnoni, 1997; Barbi, 2000; Rinaldi and Vasta, 2005; Bertoni and Randone, 2006; Ciocca, 2007; Santella, et al., 2009), with relatively stable dynamics over time (Vasta and Baccini, 1997; Rinaldi and Vasta, 2005; 2012; Santella, Drago, and Polo, 2009; Bellenzier and Grassi, 2014).

The Italian legal system does not impose prohibitions on the sharing of board members, limits on the number of different appointments that a director can hold, or restrictions on board size. The only exception is Law 214 of the year 2011, which forbids organisations from sharing board members with companies or groups operating in the banking, insurance, and financial services sectors. Lastly, a director needs her original board's approval to join the board of a competitor (Article 2390 of the Italian Civil Code).

\subsection{Specification of the network formation game}

In this section we specify the network formation game for the empirical illustration at hand. There are $N_{m} \in \mathbb{N} \backslash\{1,2\}$ firms within each industry $m \in\{1, \ldots, M\}$. The firms connect to each other by allowing their executives to sit on competitors' boards. $G_{i j}$ is equal to 1 if an executive of firm $i$ sits on firm $j$ 's board. Links are nonreciprocal: $G_{i j}$ can be different from $G_{j i}$ because $i$ having an executive sitting on $j$ 's board does not imply $j$ having an executive sitting on $i$ 's board. The consent of both $i$ and $j$ is necessary for the formation of the link $i j$. When $G_{i j}=1$, we assume that $i$ and $j$ cannot transfer utility.

Firms $i$ and $j$ are not considered connected when they share a director with non-executive 
duties at both such firms. Indeed, the literature suggests that only ties involving executive powers can represent long term economic and institutional relations between companies. Instead, non-executive directors often sit temporarily on multiple boards because of their technical skills, rather than to transfer information across firms (Mizruchi and Bunting, 1981; Stokman, Wasseur, and Elsas, 1985; Stokman, Van Der Knoop, and Wasseur, 1988). With this assumption, we want to remain conservative on the amount of information channels among firms incorporated in the analysis. If more information channels are present in reality (e.g., via the sharing of non-executive directors or via equity participations), then our results can be interpreted as underestimated.

Figure 7 displays an example of network arising in an industry composed by five firms. On the left, the board composition of every firm is indicated by two sets of letters. Each letter denotes a board member. The first set of letters is the set of executives. The second set of letters is the set of non-executives. The picture on the right shows how the network is constructed.
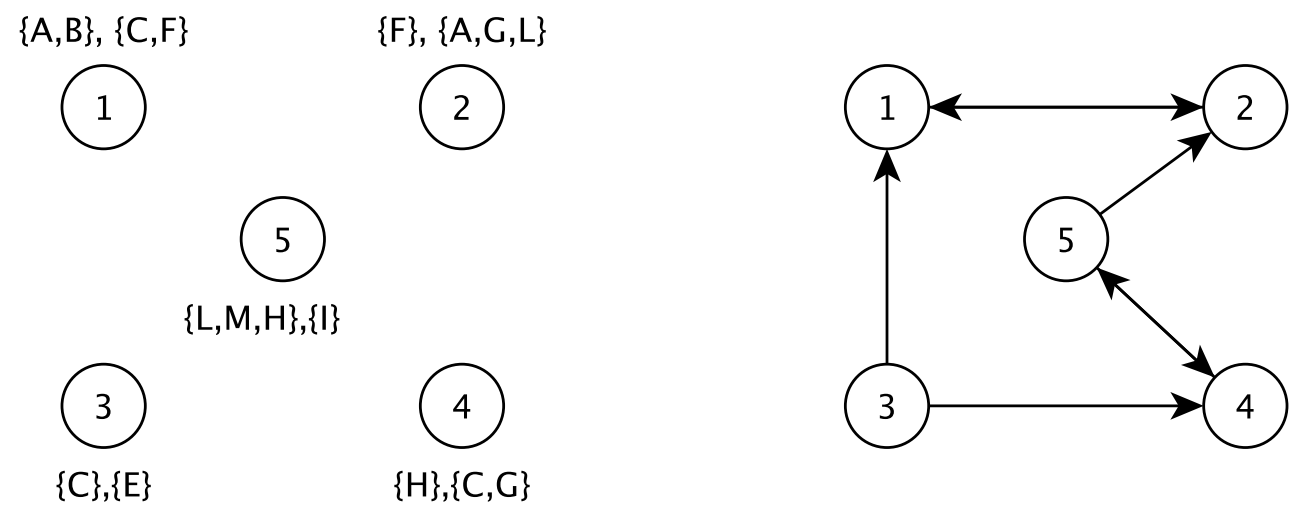

Figure 7: This figure reports an example of network arising in an industry composed by five firms. On the left, the board composition of every firm is indicated by two sets of letters. Each letter denotes a board member. The first set of letters is the set of executives. The second set of letters is the set of non-executives. The picture on the right shows how the network is constructed.

We assume that an executive of firm $i$ represents $i$ 's will when participating in firm $j$ 's board meetings with non-executive duties. We also assume that an executive of $i$ carries on $i$ and $j$ 's wills when participating in $j$ 's board meetings with executive duties. Hence, we ignore directors' identities and interpret " $G_{i j}=1, G_{j i}=0$ " as "company $i$ attends company $j$ 's board meetings, company $j$ does not attend company $i$ 's board meetings" and " $G_{i j}=1, G_{j i}=1$ " as "company $i$ attends company $j$ 's board meetings, company $j$ attends company $i$ 's board meetings".

In accordance with corporate laws, when $G_{i j}=1$, firms $i$ and $j$ interact with different roles. In particular, $i$ has the right to learn everything about $j$ 's decision making process and it is supposed to monitor and inspire $j$ 's conduct for the good of the company. Conversely, $j$ can benefit from $i$ 's guidance. Along these lines, the literature suggests various scenarios that could underlie the formation of the link ij (Selznick, 1949; Thompson and McEwen, 1958; Dooley, 1969; Allen, 1974; Bunting, 1976; Carrington, 1981; Mizruchi, 1996). Specifically, from the point of view of firm $i$, on one hand, supervising the decision making process of smaller and less profitable competitors may be favoured because smaller and less profitable competitors 
are more likely to undertake ambitious strategies with the objective of growing but potentially destabilising the industry's pace. On the other hand, according to a "learning from better practices" perspective, $i$ could prefer sitting on boards of directors of bigger and more profitable competitors. Additionally, $i$ may want to join boards targeted also by other competitors, in order to promote meetings with as many industry actors as possible and, in turn, facilitate information flow. Conversely, from the point of view of firm $j$, on one hand, hosting executives of smaller and less profitable companies may be favoured because $j$ might feel more vulnerable and controlled if exposed to organisations playing a leading role in the industry. On the other hand, hosting executives of bigger and more profitable competitors could represent a source of inspiring guidance and new opportunities.

Our methodology can be used to shed light on such conjectures by modelling firm $i$ 's payoff as

$$
\begin{aligned}
& \text { Payoff that firm } i \text { gets } \\
& \text { from attending } \\
& \text { firm } j \text { 's board meetings } \\
& u_{i}\left(\boldsymbol{G}, \boldsymbol{X}, \epsilon ; \theta_{u}\right) \equiv \sum_{j \in \mathcal{N} \backslash\{i\}} G_{i j} \times[\overbrace{r_{i j}(\boldsymbol{X}, \epsilon ; \lambda)+v_{j}\left(\sum_{k \in \mathcal{N} \backslash\{i\}} G_{k j} ; \delta\right)}]+ \\
& \sum_{j \in \mathcal{N} \backslash\{i\}} G_{j i} \times[\underbrace{l_{j i}(\boldsymbol{X}, \epsilon ; \gamma)}_{\begin{array}{c}
\text { Payoff that firm } i \text { gets } \\
\text { when firm } j
\end{array}}], \\
& \text { attends } i \text { 's board meetings }
\end{aligned}
$$

where $\boldsymbol{X}$ collects the firms' covariates, such as size and profitability, $\epsilon \equiv\left(\epsilon_{i} \forall i \in \mathcal{N}\right), \epsilon_{i} \equiv\left(\eta_{i}, \zeta_{i}\right)$, $\eta_{i} \equiv\left(\eta_{i j} \forall j \in \mathcal{N} \backslash\{i\}\right), \zeta_{i} \equiv\left(\zeta_{j i} \forall j \in \mathcal{N} \backslash\{i\}\right), \eta_{i j} \in \mathbb{R}$ is $i$ 's taste shock for the link $i j, \zeta_{j i} \in \mathbb{R}$ is $i$ 's taste shock for the link $j i, \theta_{u} \equiv(\lambda, \delta, \gamma)$, and $v_{j}(\cdot ; \delta)$ is monotone in $\sum_{k \in \mathcal{N} \backslash\{i\}} G_{k j} .{ }^{30}$

Lastly, note that cases in which firms share board members with companies operating in different industries, i.e., vertical ties, are not taken into account. In fact, before the year $2011,{ }^{31}$ in Italy vertical ties are mostly driven by connections with financial institutions, possibly arising because experts in the financial industry are useful to firms operating in other industries, or because lending banks want to control debtors' boards. The study of such financial links may require a payoff structure more sophisticated than (9) and it is outside the scope of our paper. Additionally, the current policy debate focuses on incentives behind the existence of ties within industries, rather than across industries, given the potential impact on competition.

\subsection{Data}

The sources of data are the Registro Imprese and the Cerved databases, whose access has been provided by the Bank of Italy. The Registro Imprese is a database in which all Italian companies are required to enrol through the Chamber of Commerce in their territorial province and is the primary source of certification of their constituent data. It offers accurate and updated information on individual firms (e.g., legal status, year of registration, composition of governance

\footnotetext{
${ }^{30}$ Note that $G_{j i}=1$ does not imply that $i$ can interact with the competitors participating in $j$ 's board meetings. This is why $v_{j}\left(\sum_{k \in \mathcal{N} \backslash\{i\}} G_{k j} ; \delta\right)$ is absent from $l_{j i}(\cdot ; \gamma)$. Also, we assume away any dependence of $l_{j i}(\cdot ; \gamma)$ on $\sum_{k \in \mathcal{N} \backslash\{j\}} G_{k i}$, such as a cost term depending on the total number of incoming links that firm $i$ has already formed.

${ }^{31}$ That is, prior to the time this was forbidden by Law 214 of 2011 . Note that our study uses data of 2010, as explained in Section 4.3.
} 
bodies, geographical location, and principle line of activity) and on important changes related to their existence (e.g., termination, liquidation, bankruptcy, and mergers and acquisitions). The Cerved database contains information useful for measuring the credit risk of Italian companies and provides balance sheet details.

Our sample collects all Italian joint stock companies with a governance organised under the Articles 2380-2409 of the Italian Civil Code (Societá per Azioni con sistema tradizionale) and whose data for the year 2010 were available, i.e., 2599 firms operating in $M=386$ industries.

The board composition and the role of each director (executive or non-executive) are extracted from the Registro Imprese database. Industries are constructed considering the firms' principal lines of activity provided by the 5 digit-ATECO 2002 code, also obtained from the Registro Imprese database. The ATECO 2002 code is similar to the SIC code in the UK and US. It is unique for each firm.

The existing empirical studies on board interlocks measure firm size and profitability in various ways. We measure firm size using total assets (hereafter, TA), as per Dooley (1969), Allen (1974), and Mizruchi and Stearns (1988; 1994). We measure firm profitability using return on equity (hereafter, ROE), as per Baysinger and Butler (1985) and Fligstein and Brantley (1992). Our choice to measure firm size and profitability using TA and ROE is mainly driven by data quality considerations. TA and ROE are extracted from the Cerved database. Additional control variables are not included to keep the inference part tractable.

More details on data, cleaning steps, and descriptive statistics are in Appendix ?? of the supplementary material.

\subsection{Results}

In this section we construct a $95 \%$ confidence region for the identified set characterised in Section 3 using the data described above. As the observed networks have up to 15 players, we focus on the outer set $\Theta^{\circ}$, rather on the sharp identified set. To simplify the computation, TA and ROE are discretised into five separate bins, according to their 20-th, 40-th, ..., 80-th quantiles. The inference method developed by Andrews and Soares (2010) is implemented, as illustrated in Appendix ?? of the supplementary material.

Before presenting the results, let us add few clarifying remarks. First, the number of players, $N$, varies across the observed networks. In particular, the realisations of $N$ observed in the data are $\{3,4, \ldots, 15\}$. Hence, $N$ is treated as a covariate and the moment inequalities involve the empirical probability distribution of $\boldsymbol{G}$ conditional on both $N$ and $\boldsymbol{X}$. Second, given a candidate parameter value, the number of moment inequalities to check for each observed realisation of the covariates, $(n, \boldsymbol{x})$, is $2 n \times 2^{n-1}$. Third, the firms' identities vary across the observed networks and the firms have no specific roles. Thus, the labels are assigned arbitrarily to the firms within each network. As discussed in Section 3.3, when doing inference and replacing probabilities with sample analogues, the researcher needs to ensure that the label assignment does not affect estimates. We achieve this by imposing sufficient conditions such that the probability distribution of $\boldsymbol{G}$ conditional on $(N, \boldsymbol{X})$ is invariant under permutations of the labels, as outlined by Sheng (2016). More details are in Appendix ?? of the supplementary material. 
We consider the following payoff specification:

$$
\begin{aligned}
u_{i}\left(\boldsymbol{G}, \boldsymbol{X}, \epsilon ; \theta_{u}\right) \equiv & \sum_{j \in \mathcal{N} \backslash\{i\}} G_{i j} \times\left[\lambda_{0}+\lambda_{1} \times\left(\mathrm{TA}_{j}-\mathrm{TA}_{i}\right)+\lambda_{2} \times\left(\mathrm{ROE}_{j}-\mathrm{ROE}_{i}\right)+\delta \times \sum_{k \in \mathcal{N} \backslash\{i\}} G_{k j}+\eta_{i j}\right]+ \\
& \sum_{j \in \mathcal{N} \backslash\{i\}} G_{j i} \times\left[\gamma_{0}+\gamma_{1} \times\left(\mathrm{TA}_{i}-\mathrm{TA}_{j}\right)+\gamma_{2} \times\left(\mathrm{ROE}_{i}-\mathrm{ROE}_{j}\right)+\zeta_{j i}\right] .
\end{aligned}
$$

Regarding the structure of the taste shocks, we define $\eta_{i j}$ (i.e, firm $i$ 's taste shock for attending firm $j$ 's board meetings) and $\zeta_{i j}$ (i.e., firm $j$ 's taste shock for allowing firm $i$ to attend $j$ 's board meetings) as

$$
\eta_{i j} \equiv \tau_{i j}+\alpha_{j}, \zeta_{i j} \equiv \omega_{i j}+\phi_{j} \forall(i, j) \in \mathcal{N}^{2} .
$$

$\alpha_{j}, \phi_{j}$ represent some firm $j$-specific unobserved heterogeneity that all the agents take into account when playing the section $j$ game. Note that $\alpha_{j}$ enters $\eta_{i j} \forall i \in \mathcal{N} \backslash\{j\}$ and $\phi_{j}$ enters $\zeta_{i j} \forall i \in \mathcal{N} \backslash\{j\}$. In line with Assumption 3 a), this allows for link target-specific unobserved heterogeneity but not for link source-specific unobserved heterogeneity. ${ }^{32}$ All the latent terms

\begin{tabular}{|c|c|}
\hline$\lambda_{0}$ & $10.870177 .240]$ \\
\hline$\lambda_{1}$ & $-128.453,-24.637]$ \\
\hline$\lambda_{2}$ & {$[-413.811,-143.719]$} \\
\hline$\delta$ & {$[58.060,149.446]$} \\
\hline$\gamma_{0}$ & {$\left[\begin{array}{ll}-240.590, & -99.976\end{array}\right]$} \\
\hline$\gamma_{1}$ & {$[30.044,145.750]$} \\
\hline$\gamma_{2}$ & {$[23.288,208.275]$} \\
\hline
\end{tabular}
are i.i.d. standard normals independent of $(N, \boldsymbol{X})$.

Table 1 reports the hypercube that contains the $95 \%$ confidence region for each $\theta \in \Theta^{\circ} .{ }^{33}$

Table 1: This table reports the projections of the $95 \%$ confidence region for each $\theta \in \Theta^{\circ}$ according to model specification (10).

The sign of various effects, as measured by projections of this hypercube, is analysed first. The projection for the parameter $\delta$ is $[58.060,149.446]$. The positive sign reveals that, all else equal, firm $i$ 's payoff from attending firm $j$ 's board meetings increases with the number of other rival companies participating in $j$ 's board assemblies. The projections for the parameters $\lambda_{1}$ and $\lambda_{2}$ are, respectively, $[-128.453,-24.637]$ and $[-413.811,-143.719]$, and indicate that, all else equal, $i$ prefers to attend $j$ 's board meetings when $j$ is smaller and less profitable than $i$. The projections for the parameters $\gamma_{1}$ and $\gamma_{2}$ are, respectively, [30.044, 145.750] and $[23.288,208.275]$ and suggests that, all else equal, $j$ prefers to host $i$ on its board when $i$ is smaller and less profitable than $j$.

The signs of the projections shed some light on the qualitative predictions proposed in the literature and discussed in Section 4.2. In particular, the negative signs of $\lambda_{1}$ and $\lambda_{2}$ support the idea that supervising the decision making process of smaller and less profitable competitors may be favoured, because they are more likely to undertake ambitious strategies with the objective of growing but potentially destabilising the industry's pace. The positive

\footnotetext{
${ }^{32}$ Results for the case including link source-specific unobserved heterogeneity are similar and not reported.

${ }^{33}$ This implies that the reported intervals may be too conservative. At the same time, such excess of conservativeness suggests that the results are robust in terms of the sign of various effects.
} 
signs of $\gamma_{1}$ and $\gamma_{2}$ suggest that firms may choose to host on their boards executives of smaller and less profitable companies because firms perceive themselves more vulnerable and controlled if exposed to organisations playing a leading role in the industry. Lastly, the positive sign of $\delta$ confirms that, when firms decide whether to allow their executives to join rivals' boards, they may prefer companies targeted also by other competitors, in order to promote meetings with as many industry actors as possible and, in turn, facilitate information flow.

To examine the magnitude of results, one may study how some network summary statistics (e.g., density, average degree, percentage of isolated nodes, and number of links) vary as a consequence of changes in TA or ROE. Various experiments are possible. As an example (hereafter, Experiment I), Table 2 reports the outcome of the following procedure. Within each industry, we merge the firms with TA value falling in the 20-th quantile into one new firm with TA and ROE equal to the sum of TA and ROE across the merged firms. We draw several realisations of the taste shocks. For each drawn realisation of the taste shocks and for each parameter value in the $95 \%$ confidence region, we find the set of equilibrium networks. We compute the density, the average degree, the percentage of isolated nodes and the total number of links in every equilibrium network, and record their minimum and maximum values across equilibrium networks. Then, we average the bounds across the drawn realisations of taste shocks and industries. Finally, we report the smallest lower bound and the largest upper bound across parameter values in the second and third columns of Table 2. The same experiment is repeated without merging companies and the results appear in the fourth and sixth columns of Table 2. The observed empirical values are in the fifth column of Table 2. As a consequence of the simulated shift, the upper bounds on density, average degree, and number of links decrease, while the lower bound on the percentage of isolated nodes increases. Hence, when merging the smallest firms within each industry (and thus impeding them from creating PHBIs among each other), networks can have more isolated nodes and become more disconnected.

\begin{tabular}{|c|c|c|c|c|c|}
\hline & $\begin{array}{c}\text { New } \\
\text { lower bound }\end{array}$ & $\begin{array}{c}\text { New } \\
\text { upper bound }\end{array}$ & $\begin{array}{c}\text { Old } \\
\text { lower bound }\end{array}$ & $\begin{array}{c}\text { Empirical } \\
\text { value }\end{array}$ & $\begin{array}{c}\text { Old } \\
\text { upper bound }\end{array}$ \\
\hline Density & 0 & 0.102 & 0 & 0.005 & 0.108 \\
\hline Average Degree & 0 & 0.789 & 0 & 0.022 & 0.940 \\
\hline \% Isolated nodes & 57.174 & 100 & 55.031 & 97.666 & 100 \\
\hline Number of links & 0 & 7.821 & 0 & 0.163 & 10.375 \\
\hline
\end{tabular}

Table 2: This table reports the bounds on some network summary statistics when Experiment I is run.

As an alternative experiment, we analyse the impact on firms' payoffs of a policy preventing the biggest firms within each industry from forming links. Specifically, the following procedure is implemented. Within each industry, we delete the firms with a TA value falling in the 60-th and 80 -th quantiles. We draw several realisations of the taste shocks. For each drawn realisation of the taste shocks and for each parameter value in the $95 \%$ confidence region, we find the set of equilibrium networks. We compute the average of the firms' payoffs and record its minimum and maximum values across the equilibrium networks. Then, we average the bounds across the drawn realisations of taste shocks and industries. Finally, we save the smallest lower bound and the largest upper bound across parameter values. The same experiment is repeated without 
deleting any firm. As a consequence of the simulated intervention, we find that the largest upper bound is $71.848 \%$ lower than in the original setting. This reveals that companies may be on average worse off if the largest firms are not allowed to create PHBIs.

\section{Conclusions}

We study a static game of network formation featuring complete information, nonreciprocal links, and a relatively small number of players. The payoff that player $i$ gets from linking to player $j$ is allowed to depend on the number of other players linking to $j$. Economic theory suggests that this spillover effect has a key role in many applications of interest, e.g., PHBIs, hyperlinks among news websites, advice-seeking ties, and trading networks. As the game admits multiple equilibria, we provide partial identification arguments for the payoff parameters, under the assumption that the researcher has data on several equilibrium networks. The structure assigned to the players' preferences is leveraged to simplify the problem. In particular, the spillover effect considered permits us to decompose the network formation game into some local games such that the network formation game is in equilibrium if and only if each local game is in equilibrium. This if and only if relation reveals that the set of equilibria of the network formation game is the Cartesian product of the set of equilibria of the local games, which thereby substantially decreases the curse of dimensionality in the analysis. The methodology developed is used to learn about Italian firms' incentives for forming PHBIs. We find that, all else equal, firm $i$ 's payoff from having an executive sitting on competitor $j$ 's board increases with the number of other rival companies having an executive appointed by $j$. Such a result confirms that the creation of PHBIs is motivated by information sharing purposes, as conjectured in the literature.

There are several directions to take for future research. For example, it may be of interest to investigate whether it is possible to characterise a general class of games satisfying the if and only if property or to remove the parametric distributional assumption on the latent variables.

Acknowledgements: I thank Christian Bontemps, Federico Bugni, Andrew Chesher, Allan Collard-Wexler, Alan Crawford, Martin Cripps, Aureo de Paula, Alfred Galichon, Eric Gautier, Matthew Gentry, Koen Jochmans, Jihyun Kim, Toru Kitagawa, Jia Li, Thierry Magnac, Matthew Masten, Nour Meddahi, Konrad Mierendorff, Francesca Molinari, Lars Nesheim, Elena Pastorino, Franco Peracchi, Imran Rasul, Adam Rosen, Shruti Sinha, Martin Weidner, Daniel Yi Xu, and seminar participants at the 5th European Conference on Networks. 


\section{A Weaker sufficient conditions for Proposition 1}

Proposition 1 holds also under a weaker version of Assumption 1.

Assumption $\mathbf{1}^{\prime}$. (Spillover effect - weak)

a) $u_{i}\left(\cdot ; \theta_{u}\right)$ is additively separable in player $i$ 's outgoing links, i.e.,

$$
u_{i}\left(\boldsymbol{G}, \boldsymbol{X}, \epsilon ; \theta_{u}\right) \equiv \sum_{j \in \mathcal{N} \backslash\{i\}} G_{i j} \times u_{i j}\left(\boldsymbol{G}_{-\{i \cdot\}}, \boldsymbol{X}, \epsilon ; \theta_{u}\right)
$$

where $u_{i j}\left(\cdot ; \theta_{u}\right)$ is the payoff that $i$ gets from forming the link $i j$.

b) Only player $j$ 's incoming links affect $u_{i j}\left(\cdot ; \theta_{u}\right)$, i.e.,

$$
u_{i j}\left(\boldsymbol{G}_{-\{i \cdot\}}, \boldsymbol{X}, \epsilon ; \theta_{u}\right) \equiv u_{i j}\left(\left(G_{k j} \forall k \in \mathcal{N} \backslash\{i, j\}\right), \boldsymbol{X}, \epsilon ; \theta_{u}\right) .
$$

\section{B The bilateral case}

This section explains how all the results of Section 3 can be replicated for the bilateral case. We remind the reader that, even if the formation of links is now treated as a bilateral agreement process, the focus remains on nonreciprocal links, i.e., $G_{i j}$ can be different from $G_{j i}$ for any $(i, j) \in \mathcal{N}^{2}$.

\section{B.1 The bilateral case with non-transferable payoffs}

Each player $i \in \mathcal{N}$ is endowed with some predetermined characteristics, $\left(X_{i}, \epsilon_{i}\right) . \quad X_{i} \in X \subseteq$ $\mathbb{R}^{K}$ is observed by the researcher. $\epsilon_{i} \equiv\left(\eta_{i}, \zeta_{i}\right) \in \mathbb{R}^{2(N-1)}$ is unobserved by the researcher. $\eta_{i} \equiv\left(\eta_{i j} \forall j \in \mathcal{N} \backslash\{i\}\right) \in \mathbb{R}^{N-1}$, where $\eta_{i j}$ represents player $i$ 's taste shock for the link $i j$. $\zeta_{i} \equiv\left(\zeta_{j i} \forall j \in \mathcal{N} \backslash\{i\}\right) \in \mathbb{R}^{N-1}$, where $\zeta_{j i}$ represents player $i$ 's taste shock for the link $j i . \boldsymbol{X} \equiv$ $\left(X_{i} \forall i \in \mathcal{N}\right)$ and $\epsilon \equiv\left(\epsilon_{i} \forall i \in \mathcal{N}\right)$ are common knowledge to all the players. The payoff that player $i$ gets from participating in the game depends on $(\boldsymbol{G}, \boldsymbol{X}, \epsilon)$ and is denoted by $u_{i}\left(\boldsymbol{G}, \boldsymbol{X}, \epsilon ; \theta_{u}\right)$, where $\theta_{u} \in \Theta_{u} \subseteq \mathbb{R}^{d_{u}}$ is a vector of parameters. The players announce the desired outgoing and incoming links. Links form if they are mutually beneficial. The equilibrium concept is pairwise stability (Jackson and Wolinsky, 1996). $\boldsymbol{G} \in \mathscr{G}$ is a pairwise stable (hereafter, PS) network if it is robust to unilateral one-link deletion and bilateral one-link formation. ${ }^{34}$ Utility transfers are not allowed.

\footnotetext{
${ }^{34}$ One may want to use an equilibrium concept stronger than pairwise stability, e.g., pure strategy pairwise Nash stability. In our model, pairwise stability and pure strategy pairwise Nash stability coincide.

More precisely, pure strategy pairwise Nash stable networks are robust to unilateral multi-link deletion and bilateral one-link formation (Jackson and Wolinski, 1996; Calvó-Armengol, 2004; Bloch and Jackson, 2005, 2006; Goyal and Joshi, 2006; Calvó-Armengol and Ilkiliç, 2009). Therefore, the set of pure strategy pairwise Nash stable networks is the intersection between the set of Nash stable networks (constructed by letting players announce the desired outgoing and incoming links, according to PSNE, and, then, forming the mutually announced links) and the set of PS networks. Gilles and Sarangi (2005) show that if payoffs are network convex (as in our model under Assumption B.1), then the set of PS networks coincides with the set of pure strategy pairwise Nash stable networks.
} 
Definition B.1. (PS network without transfers) $\boldsymbol{G} \in \mathscr{G}$ is a PS network without transfers if

$$
G_{i j}=\mathbb{1}\left\{\Delta_{i j}^{i}\left(\boldsymbol{G}_{-\{i j\}}, \boldsymbol{X}, \epsilon ; \theta_{u}\right) \geq 0\right\} \times \mathbb{1}\left\{\Delta_{i j}^{j}\left(\boldsymbol{G}_{-\{i j\}}, \boldsymbol{X}, \epsilon ; \theta_{u}\right) \geq 0\right\} \forall(i, j) \in \mathcal{N}^{2},
$$

where

$$
\begin{aligned}
& \Delta_{i j}^{i}\left(\boldsymbol{G}_{-\{i j\}}, \boldsymbol{X}, \epsilon ; \theta_{u}\right) \equiv u_{i}\left(\left(1, \boldsymbol{G}_{-\{i j\}}\right), \boldsymbol{X}, \epsilon ; \theta_{u}\right)-u_{i}\left(\left(0, \boldsymbol{G}_{-\{i j\}}\right), \boldsymbol{X}, \epsilon ; \theta_{u}\right), \\
& \Delta_{i j}^{j}\left(\boldsymbol{G}_{-\{i j\}}, \boldsymbol{X}, \epsilon ; \theta_{u}\right) \equiv u_{j}\left(\left(1, \boldsymbol{G}_{-\{i j\}}\right), \boldsymbol{X}, \epsilon ; \theta_{u}\right)-u_{j}\left(\left(0, \boldsymbol{G}_{-\{i j\}}\right), \boldsymbol{X}, \epsilon ; \theta_{u}\right),
\end{aligned}
$$

are the payoffs that players $i$ and $j$ receive when forming the link $i j$ respectively, $\boldsymbol{G}_{-\{i j\}}$ denotes the matrix $\boldsymbol{G}$ without $G_{i j},\left(1, \boldsymbol{G}_{-\{i j\}}\right)$ is the matrix $\boldsymbol{G}$ with $G_{i j}=1$, and $\left(0, \boldsymbol{G}_{-\{i j\}}\right)$ is the matrix $\boldsymbol{G}$ with $G_{i j}=0$.

Assumption 1 can be reformulated as follows.

Assumption B.1. (Spillover effect)

a) $u_{i}\left(\cdot ; \theta_{u}\right)$ is additively separable in player $i$ 's outgoing and incoming links, i.e.,

$$
u_{i}\left(\boldsymbol{G}, \boldsymbol{X}, \epsilon ; \theta_{u}\right) \equiv \sum_{j \in \mathcal{N} \backslash\{i\}} G_{i j} \times w_{i j}\left(\boldsymbol{G}_{-\{i, \cdot i\}}, \boldsymbol{X}, \epsilon ; \theta_{u, 1}\right)+\sum_{j \in \mathcal{N} \backslash\{i\}} G_{j i} \times l_{j i}\left(\boldsymbol{G}_{-\{i, \cdot i\}}, \boldsymbol{X}, \epsilon ; \theta_{u, 2}\right),
$$

where $\boldsymbol{G}_{-\{i \cdot, i\}}$ denotes the matrix $\boldsymbol{G}$ without $i$ 's outgoing and incoming links, $w_{i j}\left(\cdot ; \theta_{u}\right)$ is the payoff that $i$ gets from forming the link $i j, l_{j i}\left(\cdot ; \theta_{u}\right)$ is the payoff that $i$ gets from forming the link $j i$, and $\theta_{u} \equiv\left(\theta_{u, 1}, \theta_{u, 2}\right)$.

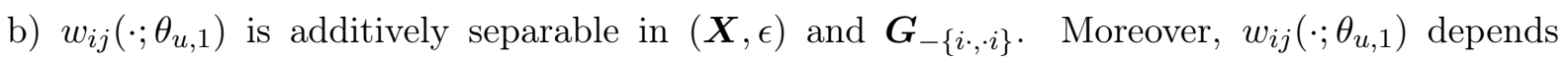
on $\boldsymbol{G}_{-\{i \cdot, i\}}$ only through the number of player $j$ 's incoming links and this dependence is monotonic. Specifically,

$$
w_{i j}\left(\boldsymbol{G}_{-\{i, \cdot i\}}, \boldsymbol{X}, \epsilon ; \theta_{u, 1}\right) \equiv r_{i j}(\boldsymbol{X}, \epsilon ; \lambda)+v_{j}\left(\sum_{k \in \mathcal{N} \backslash\{i\}} G_{k j} ; \delta\right)
$$

where $\lambda$ and $\delta$ are vectors of parameters, $\theta_{u, 1} \equiv(\lambda, \delta)$, and $v_{j}(\cdot ; \delta)$ is monotone in $\sum_{k \in \mathcal{N} \backslash\{i\}} G_{k j}$.

c) $l_{j i}\left(\cdot ; \theta_{u, 2}\right)$ does not depend on $\boldsymbol{G}_{-\{i, \cdot i\}}$, i.e.,

$$
l_{j i}\left(\boldsymbol{G}_{-\{i \cdot, i\}}, \boldsymbol{X}, \epsilon ; \theta_{u, 2}\right) \equiv l_{j i}\left(\boldsymbol{X}, \epsilon ; \theta_{u, 2}\right) .
$$

Let us make some notational remarks to avoid any misinterpretation. Under Assumption B.1, Definition B.1 becomes: $\boldsymbol{G} \in \mathscr{G}$ is a PS network if

$$
G_{i j}=\mathbb{1}\left\{r_{i j}(\boldsymbol{X}, \epsilon ; \lambda)+v_{j}\left(\sum_{k \in \mathcal{N} \backslash\{i\}} G_{k j} ; \delta\right) \geq 0\right\} \times \mathbb{1}\left\{l_{i j}\left(\boldsymbol{X}, \epsilon ; \theta_{u, 2}\right) \geq 0\right\} \forall(i, j) \in \mathcal{N}^{2}
$$

Given this definition, we remind the reader that, in equilibrium, $G_{i j}=1$ if

$$
r_{i j}(\boldsymbol{X}, \epsilon ; \lambda)+v_{j}\left(\sum_{k \in \mathcal{N} \backslash\{i\}} G_{k j} ; \delta\right) \geq 0 \text { and } l_{i j}\left(\boldsymbol{X}, \epsilon ; \theta_{u, 2}\right) \geq 0,
$$


while $G_{j i}=1$ if

$$
r_{j i}(\boldsymbol{X}, \epsilon ; \lambda)+v_{i}\left(\sum_{k \in \mathcal{N} \backslash\{j\}} G_{k i} ; \delta\right) \geq 0 \text { and } l_{j i}\left(\boldsymbol{X}, \epsilon ; \theta_{u, 2}\right) \geq 0 .
$$

As Assumption B.1 highlights, in the bilateral case the focus of our work remains on the same spillover effect as in the unilateral case. Such spillover effect has a key role in many applications of economic interest, as discussed in Section 3.1 with four examples. In particular, three out of those four examples typically feature a bilateral agreement process. These are PHBIs, advice-seeking ties, and trading networks. In all the three cases it is reasonable to assume that $l_{i j}\left(\boldsymbol{X}, \epsilon ; \theta_{u, 2}\right)$ does not depend on $v_{i}\left(\sum_{k \in \mathcal{N} \backslash\{j\}} G_{k i} ; \delta\right)$. Specifically, with regards to PHBIs, the reader can refer to Section 4.2 for explanations. With regards to advice-seeking ties, $G_{i j}=1$ if agent $i$ consults agent $j$. The payoff that $j$ gets from giving advice to $i$ is not expected to depend on $i$ 's reputation, which is proxied by the number of agents seeking $i$ for advice. With regards to trading networks, consider a group of companies each offering a different service, e.g., legal, accounting, consulting, marketing, etc. Suppose that every company has to choose between internalising and buying from other firms in the group any service needed for operational activities. $G_{i j}=1$ if firm $i$ buys firm $j$ 's service. Given that each company offers a different service, sellers do not compete for buyers. Therefore, the payoff that $j$ gets from selling to $i$ is not expected to depend on the number of additional sellers bargaining with $i$.

Lastly, Assumption B.1 excludes any dependence of $l_{i j}(\cdot ; \gamma)$ on $\sum_{k \in \mathcal{N} \backslash\{i\}} G_{k j}$, such as a cost term depending on the total number of incoming links that player $j$ has already formed.

The analysis in Section 3 can be adapted to model (B.1) after having replaced Assumption 1 with Assumption B.1 and defined $\epsilon_{i j} \equiv\left(\eta_{i j}, \zeta_{i j}\right)$.

\section{B.2 The bilateral case with transferable payoffs}

When transfers are allowed, assumptions on strategies, preferences, and information follow Section B.1. Instead, the notion of pairwise stability should be changed as follows (Bloch and Jackson, 2006).

Definition B.2. (PS network with transfers) $\boldsymbol{G} \in \mathscr{G}$ is a PS network with transfers if

$$
G_{i j}=\mathbb{1}\left\{\Delta_{i j}^{i}\left(\boldsymbol{G}_{-\{i j\}}, \boldsymbol{X}, \epsilon ; \theta_{u}\right)+\Delta_{i j}^{j}\left(\boldsymbol{G}_{-\{i j\}}, \boldsymbol{X}, \epsilon ; \theta_{u}\right) \geq 0\right\} \forall(i, j) \in \mathcal{N}^{2}
$$

The analysis in Section 3 can be adapted to model (B.5) after having replaced Assumption 1 with Assumption B.1 and defined $\epsilon_{i j} \equiv \eta_{i j}+\zeta_{i j}$.

\section{Proofs}

Proof of Lemma 1. Note that, under Assumption 1 a),

$$
G_{i j}=\mathbb{1}\left\{u_{i j}\left(\boldsymbol{G}_{-\{i \cdot\}}, \boldsymbol{X}, \epsilon ; \theta_{u}\right) \geq 0\right\}
$$


is equivalent to

$$
u_{i}\left(G_{i j}, \boldsymbol{G}_{-\{i j\}}, \boldsymbol{X}, \epsilon ; \theta_{u}\right) \geq u_{i}\left(\tilde{G}_{i j}, \boldsymbol{G}_{-\{i j\}}, \boldsymbol{X}, \epsilon ; \theta_{u}\right) \forall \tilde{G}_{i j} \in\{0,1\}
$$

and to

$$
\left(G_{i j}-\tilde{G}_{i j}\right) \times u_{i j}\left(\boldsymbol{G}_{-\{i \cdot\}}, \boldsymbol{X}, \epsilon ; \theta_{u}\right) \geq 0 \forall \tilde{G}_{i j} \in\{0,1\} .
$$

It is firstly proved that, under Assumption 1 a), if $\boldsymbol{G} \in \mathscr{G}$ is a PSNE of the network formation game, then (C.1) is satisfied $\forall(i, j) \in \mathcal{N}^{2}$. For any $(i, j) \in \mathcal{N}^{2}$, let $G_{i \cdot,-\{i j\}}$ be the vector $G_{i}$. with the $i j$-th element removed. By setting $\tilde{G}_{i} \equiv\left(\tilde{G}_{i j}, G_{i,-\{i j\}}\right)$ in $u_{i}\left(G_{i \cdot}, \boldsymbol{G}_{-\{i \cdot\}}, \boldsymbol{X}, \epsilon ; \theta_{u}\right) \geq$ $u_{i}\left(\tilde{G}_{i \cdot}, \boldsymbol{G}_{-\{i \cdot\}}, \boldsymbol{X}, \epsilon ; \theta_{u}\right)$ of Definition 1 for each $\tilde{G}_{i j} \in\{0,1\}$, it follows that (C.1) is satisfied.

Conversely, it is proved that, under Assumption 1 a), if the RHS of (C.2) holds $\forall(i, j) \in \mathcal{N}^{2}$, then $\boldsymbol{G} \in \mathscr{G}$ is a PSNE of the network formation game. For any $i \in \mathcal{N}$, if (C.2) holds $\forall j \in \mathcal{N} \backslash\{i\}$, then,

$$
\begin{gathered}
\sum_{j \in \mathcal{N} \backslash\{i\}}\left(G_{i j}-\tilde{G}_{i j}\right) \times u_{i j}\left(\boldsymbol{G}_{-\{i \cdot\}}, \boldsymbol{X}, \epsilon ; \theta_{u}\right) \geq 0 \forall \tilde{G}_{i \cdot} \equiv\left(\tilde{G}_{i j} \forall j \in \mathcal{N} \backslash\{i\}\right) \in\{0,1\}^{N-1}, \\
\sum_{j \in \mathcal{N} \backslash\{i\}} G_{i j} \times u_{i j}\left(\boldsymbol{G}_{-\{i \cdot\}}, \boldsymbol{X}, \epsilon ; \theta_{u}\right) \geq \sum_{j \in \mathcal{N} \backslash\{i\}} \tilde{G}_{i j} \times u_{i j}\left(\boldsymbol{G}_{-\{i \cdot\}}, \boldsymbol{X}, \epsilon ; \theta_{u}\right) \forall \tilde{G}_{i \cdot} \in\{0,1\}^{N-1}, \\
\mathbb{\Downarrow} \text { by Assumption } 1 \text { a }) \\
u_{i}\left(G_{i \cdot}, \boldsymbol{G}_{-\{i \cdot\}}, \boldsymbol{X}, \epsilon ; \theta_{u}\right) \geq u_{i}\left(\tilde{G}_{i \cdot}, \boldsymbol{G}_{-\{i \cdot\}}, \boldsymbol{X}, \epsilon ; \theta_{u}\right) \forall \tilde{G}_{i} \cdot \in\{0,1\}^{N-1} .
\end{gathered}
$$

Hence, by Definition $1, \boldsymbol{G} \in \mathscr{G}$ is a PSNE of the network formation game.

Proof of Proposition 1. It is firstly proved that, under Assumption 1, if $\boldsymbol{G} \equiv\left(G_{\cdot 1}, G_{\cdot 2}, \ldots, G_{\cdot N}\right) \in$ $\mathcal{G}$ is a PSNE of the network formation game, then $G_{\cdot j} \in\{0,1\}^{N-1}$ is a PSNE of the section $j$ game $\forall j \in \mathcal{N}$. By Lemma 1 , if $\boldsymbol{G}$ is a PSNE of the network formation game, then $G_{i j}=\mathbb{1}\left\{z_{i j}(\boldsymbol{X}, \epsilon ; \beta)+v_{j}\left(\sum_{k \in \mathcal{N} \backslash\{i\}} G_{k j} ; \delta\right) \geq 0\right\} \forall i \in \mathcal{N} \backslash\{j\}$, which is the definition of a PSNE of the section $j$ games (Definition 2). This holds $\forall j \in \mathcal{N}$.

Conversely, under Assumption 1, if $G_{\cdot j} \in\{0,1\}^{N-1}$ is a PSNE of the section $j$ game $\forall j \in \mathcal{N}$, then $\boldsymbol{G} \equiv\left(G_{\cdot 1}, G_{\cdot 2}, \ldots, G_{\cdot N}\right) \in \mathscr{G}$ is a PSNE of the network formation game. Indeed, by stacking one after the other the $N$ systems of equations characterising a PSNE of the section 1 game ..., section $N$ game, we obtain the system of equations of Lemma 1, which defines a PSNE of the network formation game.

Theorem C.1. (Tarski's fixed point theorem) Let $Q(\cdot)$ be a monotone increasing function from a non-empty complete lattice $A$ into $A$. Then, the set of fixed points of $Q(\cdot)$ in $A$ is a non-empty complete lattice.

Proof of Proposition 2. Consider any $j \in \mathcal{N}$. It is firstly discussed the case where $v_{j}(\cdot ; \delta)$ is monotone increasing. Let

$$
h_{i j}\left(G_{\cdot j}\right) \equiv \mathbb{1}\left\{z_{i j}(\boldsymbol{X}, \epsilon ; \beta)+v_{j}\left(\sum_{k \in \mathcal{N} \backslash\{i\}} G_{k j} ; \delta\right) \geq 0\right\}
$$


$\forall i \in \mathcal{N} \backslash\{j\}$ and

$$
h(G \cdot j) \equiv\left(\begin{array}{c}
h_{1 j}(G \cdot j) \\
h_{2 j}(G \cdot j) \\
\vdots \\
h_{j-1 j}\left(G_{\cdot j}\right) \\
h_{j+1}\left(G_{\cdot j}\right) \\
\vdots \\
h_{N j}\left(G_{\cdot j}\right)
\end{array}\right) .
$$

Hence, $h:\{0,1\}^{N-1} \rightarrow\{0,1\}^{N-1}$. We now show that the sufficient conditions of Theorem C.1 are satisfied when $v_{j}(\cdot ; \delta)$ is monotone increasing, which, in turn, guarantees existence of a PSNE of the section $j$ game. Let the comparison between vectors be coordinate-wise, i.e., for any $G_{\cdot j}, \tilde{G}_{\cdot j} \in\{0,1\}^{N-1}$,

$$
G_{\cdot j} \geq \tilde{G}_{\cdot j} \Leftrightarrow G_{i j} \geq \tilde{G}_{i j} \forall i \in \mathcal{N} \backslash\{j\}
$$

Thus, $G_{\cdot j}=\tilde{G}_{\cdot j}$ if and only if $G_{\cdot j} \geq \tilde{G}_{\cdot j}$ and $G_{\cdot j} \leq \tilde{G}_{\cdot j}$. Moreover, $G_{\cdot j}$ and $\tilde{G}_{\cdot j}$ are unordered if and only if neither $G_{\cdot j} \geq \tilde{G}_{\cdot j}$ nor $G_{\cdot j} \leq \tilde{G}_{\cdot j}$. Therefore, $\{0,1\}^{N-1}$ is a lattice, i.e, a set with a partial order. As $\{0,1\}^{N-1}$ is a finite lattice, it is complete. Furthermore, if $v_{j}(\cdot ; \delta)$ is monotone increasing, then $h$ is a monotone increasing function. In fact, consider two vectors $G_{. j} \geq \tilde{G}_{\cdot j}$. Since $G_{i j} \geq \tilde{G}_{i j} \forall i \in \mathcal{N} \backslash\{j\}$, then $z_{i j}(\boldsymbol{X}, \epsilon ; \beta)+v_{j}\left(\sum_{k \in \mathcal{N} \backslash\{i\}} G_{k j} ; \delta\right) \geq z_{i j}(\boldsymbol{X}, \epsilon ; \beta)+$ $v_{j}\left(\sum_{k \in \mathcal{N} \backslash\{i\}} \tilde{G}_{k j} ; \delta\right) \forall i \in \mathcal{N} \backslash\{j\}$. Hence, $h\left(G_{\cdot j}\right) \geq h\left(\tilde{G}_{\cdot j}\right)$ and the sufficient conditions of the theorem are met.

Consider the case where $v_{j}(\cdot ; \delta)$ is monotone decreasing. As explained in Section 3.2, the section $j$ game when $v_{j}(\cdot ; \delta)$ is monotone decreasing is similar in structure to an entry game with substitution effects. Existence of a PSNE in an entry game with substitution effects is proved by Berry (1992) through a constructive proof which can be reinterpreted for the section $j$ game as follows. Let $Y_{i j} \equiv z_{i j}(\boldsymbol{X}, \epsilon ; \beta)$. The elements in the sequence $\left\{Y_{i j}\right\}_{\forall i \in \mathcal{N} \backslash\{j\}}$ are ordered from largest to smallest. Let $k \in\{1, \ldots, N-1\}$ denote the position of $Y_{i j}$ in the ordered list and let $\pi$ be a function such that $\pi(i j)=k, \forall i \in \mathcal{N} \backslash\{j\}$. By replacing the subscript $i j$ with $k$, the ordered sequence is

$$
Y_{1} \geq Y_{2} \geq \ldots \geq Y_{k} \geq \ldots \geq Y_{N-1}
$$

Let $Y_{0} \equiv \max \left\{Y_{1},-v_{j}(-1 ; \delta)\right\} . \quad n_{j}^{*}$ is defined as the largest element of the set of integers $\{0,1, \ldots, N-1\}$ satisfying $Y_{n_{j}^{*}}+v_{j}\left(n_{j}^{*}-1 ; \delta\right) \geq 0$, i.e.

$$
n_{j}^{*} \equiv \max \left\{k \in\{0, \ldots, N-1\} \mid Y_{k}+v_{j}(k-1 ; \delta) \geq 0\right\} .
$$

Consider $G_{\cdot j}$ with $G_{i j}=1$ if $\pi(i j) \leq n_{j}^{*}$ and $G_{i j}=0$ otherwise. One can see that $G_{\cdot j}$ is a PSNE 
of the section $j$ game. In fact, given the definition of $n_{j}^{*}$,

$$
\begin{aligned}
Y_{0}+v_{j}(-1 ; \delta) \geq 0 & (\mathrm{a}), \\
Y_{1}+v_{j}(0 ; \delta) \geq 0 & (\mathrm{~b}), \\
& \ldots \\
Y_{n_{j}^{*}}+v_{j}\left(n_{j}^{*}-1 ; \delta\right) \geq 0 & (\mathrm{c}), \\
Y_{n_{j}^{*}+1}+v_{j}\left(n_{j}^{*} ; \delta\right)<0 & (\mathrm{~d}), \\
Y_{n_{j}^{*}+2}+v_{j}\left(n_{j}^{*}+1 ; \delta\right)<0 & (\mathrm{e}), \\
& \ldots \\
Y_{N-1}+v_{j}(N-2 ; \delta)<0 & \text { (f). }
\end{aligned}
$$

We know that $G_{. j}$ is a PSNE of the section $j$ game iff the following inequalities are satisfied

$$
\begin{array}{rc}
Y_{1}+v_{j}\left(n_{j}^{*}-1 ; \delta\right) \geq 0 & (\mathrm{~g}), \\
Y_{2}+v_{j}\left(n_{j}^{*}-1 ; \delta\right) \geq 0 & (\mathrm{~h}), \\
& \ldots \\
Y_{n_{j}^{*}}+v_{j}\left(n_{j}^{*}-1 ; \delta\right) \geq 0 & (\mathrm{i}), \\
Y_{n_{j}^{*}+1}+v_{j}\left(n_{j}^{*} ; \delta\right)<0 & (\mathrm{l}), \\
Y_{n_{j}^{*}+2}+v_{j}\left(n_{j}^{*} ; \delta\right)<0 & (\mathrm{~m}), \\
& \ldots \\
Y_{N-1}+v_{j}\left(n_{j}^{*} ; \delta\right)<0 & (\mathrm{n}) .
\end{array}
$$

By observing that inequalities (g), (h), ...,(i) are implied by inequality (c) - as $Y_{1} \geq Y_{2} \geq \ldots \geq$ $Y_{n_{j}^{*}}$ - and all the other inequalities follow from inequality (d) - as $Y_{n_{j}^{*}+1} \geq Y_{n_{j}^{*}+2} \geq \ldots \geq Y_{N-1}$ -, it can be concluded that $G_{. j}$ is a PSNE of the section $j$ game.

For the bilateral case with non-transferable payoffs illustrated in Section B.1, one can proceed analogously after having changed the equilibrium notion and imposed

$$
h_{i j}\left(G_{\cdot j}\right) \equiv \mathbb{1}\left\{r_{i j}(\boldsymbol{X}, \epsilon ; \lambda)+v_{j}\left(\sum_{k \in \mathcal{N} \backslash\{i\}} G_{k j} ; \delta\right) \geq 0\right\} \times \mathbb{1}\left\{l_{i j}\left(\boldsymbol{X}, \epsilon ; \theta_{u, 2}\right) \geq 0\right\},
$$

$\forall i \in \mathcal{N} \backslash\{j\}$ when $v_{j}(\cdot ; \delta)$ is monotone increasing, and

$$
Y_{i j} \equiv \begin{cases}r_{i j}(\boldsymbol{X}, \epsilon ; \lambda) & \text { if } l_{i j}\left(\boldsymbol{X}, \epsilon ; \theta_{u, 2}\right) \geq 0 \\ -\infty & \text { otherwise, }\end{cases}
$$

$\forall i \in \mathcal{N} \backslash\{j\}$ when $v_{j}(\cdot ; \delta)$ is monotone decreasing.

Proof of Corollary 1. The first statement of Corollary 1 is a direct application of Tarski's fixed point theorem. The second statement is now shown. As in Berry (1992), take the PSNE of the section $j$ game constructed in the proof of Proposition 2. Suppose there exists another equilibrium with $k^{*}>n_{j}^{*}$ links. In this equilibrium, some of the players associated with inequal- 
ities $(\mathrm{l}),(\mathrm{m}), \ldots,(\mathrm{n})$ above should be willing to form a link pointing to player $j$. However, none of them are happy to do so because inequalities (l), (m),..., (n) imply that

$$
\begin{array}{cc}
Y_{n_{j}^{*}+1}+v_{j}\left(k^{*}-1 ; \delta\right)<0 & \left(\mathrm{l}^{\prime}\right), \\
Y_{n_{j}^{*}+2}+v_{j}\left(k^{*}-1 ; \delta\right)<0 & \left(\mathrm{~m}^{\prime}\right), \\
& \ldots \\
Y_{N-1}+v_{j}\left(k^{*}-1 ; \delta\right)<0 & \left(\mathrm{n}^{\prime}\right) .
\end{array}
$$

Therefore, there cannot be an equilibrium with $k^{*}>n_{j}^{*}$ links.

Suppose there exists another equilibrium with $k^{*}<n_{j}^{*}$ links. In this equilibrium, some of the players associated with inequalities $(\mathrm{g}),(\mathrm{h}), \ldots$, (i) above should be willing not to form a link pointing to player $j$. However, all of them will deviate, as inequalities (g), (h),.., (i) imply that

$$
\begin{array}{rc}
Y_{1}+v_{j}\left(k^{*} ; \delta\right) \geq 0 & \left(\mathrm{~g}^{\prime}\right), \\
Y_{2}+v_{j}\left(k^{*} ; \delta\right) \geq 0 & \left(\mathrm{~h}^{\prime}\right), \\
& \ldots \\
Y_{n_{j}^{*}}+v_{j}\left(k^{*} ; \delta\right) \geq 0 & \left(\mathrm{i}^{\prime}\right) .
\end{array}
$$

Therefore, that there cannot be an equilibrium with $k^{*}<n_{j}^{*}$ links.

Proof of Corollary 2. By Proposition 2, under Assumption 1, there exists a PSNE of the section $j$ game $\forall j \in \mathcal{N}$. By Proposition 1, under Assumption 1, if $G_{. j} \in\{0,1\}^{N-1}$ is a PSNE of the section $j$ game $\forall j \in \mathcal{N}$, then $\boldsymbol{G} \equiv\left(G_{\cdot 1}, G_{.2}, \ldots, G_{\cdot N}\right) \in \mathscr{G}$ is a PSNE of the network formation game. Therefore, under Assumption 1, the network formation game has a PSNE.

Proof of Proposition 3. The proof is articulated as follows: step 1 shows that, under Assumptions 1 and 2, $\Theta^{\star \star} \supseteq \Theta^{\star}$; step 2 shows that, under Assumptions 1, 2, and 3, $\Theta^{\star \star}=\Theta^{\star}$.

Step 1 We show that, under Assumptions 1 and $2, \Theta^{\star \star} \supseteq \Theta^{\star}$. Specifically, we prove that if $\theta \in \Theta$ is such that

$$
\mathbb{P}(\boldsymbol{G} \in K \mid \boldsymbol{X}=\boldsymbol{x}) \leq T_{\boldsymbol{x}, \theta}(K) \forall K \in \mathscr{K}_{\mathscr{g}}, \forall \boldsymbol{x} \in \mathscr{X}^{n} \text { a.s. }
$$

then

$$
\mathbb{P}\left(G_{\cdot j} \in K_{j} \mid \boldsymbol{X}=\boldsymbol{x}\right) \leq T_{j, \boldsymbol{x}, \theta}\left(K_{j}\right) \forall K_{j} \in \mathscr{K}_{\{0,1\}^{n-1}}, \forall j \in \mathcal{N}, \forall \boldsymbol{x} \in \mathscr{X}^{n} \text { a.s. }
$$

For any $j \in \mathcal{N}$ and $K_{j} \in \mathscr{K}_{\{0,1\}^{n-1}}$, take $K \in \mathcal{A}_{\mathscr{G}}$ corresponding to

$$
\underbrace{\{0,1\}^{n-1} \times \ldots \times\{0,1\}^{n-1}}_{j-1 \text { times }} \times K_{j} \times \underbrace{\{0,1\}^{n-1} \times \ldots \times\{0,1\}^{n-1}}_{n-j \text { times }}
$$


where $X$ is the Cartesian product operator. By Proposition 1, (C.3) is equivalent to

$$
\begin{gathered}
\mathbb{P}\left(G_{\cdot 1} \in\{0,1\}^{n-1}, \ldots, G_{\cdot j-1} \in\{0,1\}^{n-1}, G_{\cdot j} \in K_{j}, G_{\cdot j+1} \in\{0,1\}^{n-1}, \ldots, G_{\cdot n} \in\{0,1\}^{n-1} \mid \boldsymbol{X}=\boldsymbol{x}\right) \\
\leq \mathbb{P}\left(\mathcal{S}_{1, \theta_{u}}\left(\boldsymbol{X}, \epsilon_{\cdot 1}\right) \cap\{0,1\}^{n-1} \neq \emptyset, \ldots, \mathcal{S}_{j-1, \theta_{u}}\left(\boldsymbol{X}, \epsilon_{\cdot j-1}\right) \cap\{0,1\}^{n-1} \neq \emptyset, \mathcal{S}_{j, \theta_{u}}\left(\boldsymbol{X}, \epsilon_{\cdot j}\right) \cap K_{j} \neq \emptyset,\right. \\
\left.\mathcal{S}_{j+1, \theta_{u}}\left(\boldsymbol{X}, \epsilon_{\cdot j+1}\right) \cap\{0,1\}^{n-1} \neq \emptyset, \ldots, \mathcal{S}_{n, \theta_{u}}\left(\boldsymbol{X}, \epsilon_{\cdot n}\right) \cap\{0,1\}^{n-1} \neq \emptyset \mid \boldsymbol{X}=\boldsymbol{x} ; \theta\right),
\end{gathered}
$$

$\forall \boldsymbol{x} \in \mathscr{X}^{n}$ a.s., which is equivalent to

$$
\mathbb{P}\left(G_{\cdot j} \in K_{j} \mid \boldsymbol{X}=\boldsymbol{x}\right) \leq \mathbb{P}\left(\delta_{j, \theta_{u}}\left(\boldsymbol{X}, \epsilon_{\cdot j}\right) \cap K_{j} \neq \emptyset \mid \boldsymbol{X}=\boldsymbol{x} ; \theta\right),
$$

$\forall \boldsymbol{x} \in \mathscr{X}^{n}$ a.s. By repeating the same arguments $\forall K_{j} \in \mathscr{K}_{\{0,1\}^{n-1}}$ and $\forall j \in \mathcal{N}$, all the inequalities in (C.4) are obtained.

Step 2 We show that, under Assumptions 1, 2, and 3, $\Theta^{\star \star}=\Theta^{\star}$. As discussed in step 1, under Assumptions 1 and 2, if $\theta \in \Theta^{\star}$ then $\theta \in \Theta^{\star \star}$. Hence, it is left to prove that, under Assumptions 1,2 , and 3 , if $\theta \in \Theta^{\star \star}$ then $\theta \in \Theta^{\star}$.

Step 2.1 Under Assumption 2, we rewrite $\Theta^{\star}$ and $\Theta^{\star \star}$ using more handy expressions by building on results from BMM. In what follows, given a random set $\mathscr{A}, \operatorname{Sel}(\mathscr{A})$ denotes the collection of its selections.

By the definition of selection, $\boldsymbol{g}: \Omega \rightarrow \mathscr{G}$ belongs to $\operatorname{Sel}\left(\mathcal{S}_{\theta_{u}}(\boldsymbol{X}, \epsilon)\right)$ if $g(\omega) \in \mathcal{S}_{\theta_{u}}(\boldsymbol{X}(\omega), \epsilon(\omega))$ a.s. Similarly, $\boldsymbol{g}_{\cdot j}: \Omega \rightarrow\{0,1\}^{n-1}$ belongs to $\operatorname{Sel}\left(\mathcal{S}_{j, \theta_{u}}\left(\boldsymbol{X}, \epsilon_{\cdot j}\right)\right)$ if $\boldsymbol{q}_{\cdot j}(\omega) \in \mathcal{S}_{j, \theta_{u}}(\boldsymbol{X}(\omega), \epsilon \cdot j(\omega))$ a.s. ${ }^{35}$ Moreover, by Proposition 1 ,

$$
\boldsymbol{q} \equiv\left(g_{\cdot 1}, \ldots, g_{\cdot n}\right) \in \operatorname{Sel}\left(S_{\theta_{u}}(\boldsymbol{X}, \epsilon)\right) \Leftrightarrow q_{\cdot j} \in \operatorname{Sel}\left(\mathcal{S}_{j, \theta_{u}}(\boldsymbol{X}, \epsilon \cdot j)\right) \forall j \in \mathcal{N}
$$

Given the selection $\boldsymbol{g} \equiv\left(\mathcal{g}_{\cdot 1}, \ldots, \boldsymbol{q}_{\cdot n}\right) \in \operatorname{Sel}\left(\mathcal{S}_{\theta_{u}}(\boldsymbol{X}, \epsilon)\right)$ and the realisation $\boldsymbol{g} \equiv\left(g_{\cdot 1}, \ldots, g_{\cdot n}\right) \in$ $\mathscr{G}$, let $\mathscr{E}_{\theta_{u}, g}^{g} \subseteq \mathbb{R}^{n(n-1)}$ be the collection of realisations of $\epsilon$ such that the players choose to play $\boldsymbol{g}$ in the network formation game. Let $\mathscr{E}_{\theta_{u}, q \cdot j}^{g \cdot j} \subseteq \mathbb{R}^{n-1}$ be the collection of realisations of $\epsilon_{\cdot j}$ such that the players choose to play $g_{\cdot j}$ in the section $j$ game. Define

$$
q_{g} \equiv\left(\mathbb{1}\left\{\epsilon \in \mathscr{C}_{\theta_{u}, q}^{g}\right\} \forall \boldsymbol{g} \in \mathscr{G}\right)
$$

of size $2^{n(n-1)} \times 1$. Note that, by Proposition 1 ,

$$
q_{g}=\left(\Pi_{j=1}^{n} \mathbb{1}\left\{\epsilon_{\cdot j} \in \mathscr{E}_{\theta_{u}, q_{\cdot j}}^{g_{\cdot j}}\right\} \forall(g \cdot 1, \ldots, g \cdot n) \in \mathscr{G}\right) .
$$

Let

$$
\mathbb{Q}_{\theta_{u}}(\boldsymbol{X}, \epsilon) \equiv\left\{q_{g} \mid g \in \operatorname{Sel}\left(\delta_{\theta_{u}}(\boldsymbol{X}, \epsilon)\right)\right\} .
$$

$\mathbb{Q}_{\theta_{u}}(\boldsymbol{X}, \epsilon)$ can be shown to be a random closed set. As all the selections of $\mathbb{Q}_{\theta_{u}}(\boldsymbol{X}, \epsilon)$ are

\footnotetext{
${ }^{35}$ Note the difference in notation: calligraphic small letters denote selections, non-calligraphic small letters denote realisations of random variables. In particular, $\boldsymbol{g}$ is a selection of the network formation game, $\boldsymbol{g}$ is a realisation of $\boldsymbol{G} \cdot g_{\cdot j}$ is a selection of the section $j$ game, $g_{\cdot j}$ is a realisation of $G \cdot j$.
} 
integrable, one can define the conditional Aumann expectation of $\mathbb{Q}_{\theta_{u}}(\boldsymbol{X}, \epsilon)$ as

$$
\mathbb{E}\left(\mathbb{Q}_{\theta_{u}}(\boldsymbol{X}, \epsilon) \mid \boldsymbol{X}=\boldsymbol{x} ; \theta\right) \equiv\left\{E\left(q_{g} \mid \boldsymbol{X}=\boldsymbol{x} ; \theta\right) \mid q_{g} \in \operatorname{Sel}\left(\mathbb{Q}_{\theta_{u}}(\boldsymbol{X}, \epsilon)\right)\right\}
$$

$\forall \boldsymbol{x} \in \mathfrak{X}^{n}$, where $E$ denotes the "classical" expectation. Hence, $\mathbb{E}\left(\mathbb{Q}_{\theta_{u}}(\boldsymbol{X}, \epsilon) \mid \boldsymbol{X}=\boldsymbol{x} ; \theta\right)$ is the collection of the probability distributions of $\boldsymbol{G}$ conditional on $\boldsymbol{X}=\boldsymbol{x}$ predicted by the model given $\theta$.

We introduce the same objects for the local games. For each $j \in \mathcal{N}$, define

$$
q_{q \cdot j} \equiv\left(\mathbb{1}\left\{\epsilon_{\cdot j} \in \mathscr{E}_{\theta_{u}, q \cdot j}^{g \cdot j}\right\} \forall g_{\cdot j} \in\{0,1\}^{n-1}\right),
$$

of size $2^{n-1} \times 1$. Let

$$
\mathbb{Q}_{j, \theta_{u}}\left(\boldsymbol{X}, \epsilon_{\cdot j}\right) \equiv\left\{q_{q_{\cdot j}} \mid q_{\cdot j} \in \operatorname{Sel}\left(\mathcal{S}_{j, \theta_{u}}\left(\boldsymbol{X}, \epsilon_{\cdot j}\right)\right)\right\}
$$

$\mathbb{Q}_{j, \theta_{u}}\left(\boldsymbol{X}, \epsilon_{\cdot j}\right)$ can be shown to be a random closed set. As all the selections of $\mathbb{Q}_{j, \theta_{u}}\left(\boldsymbol{X}, \epsilon_{\cdot j}\right)$ are integrable, one can define the conditional Aumann expectation of $\mathbb{Q}_{j, \theta_{u}}\left(\boldsymbol{X}, \epsilon_{\cdot j}\right)$ as

$$
\mathbb{E}\left(\mathbb{Q}_{j, \theta_{u}}\left(\boldsymbol{X}, \epsilon_{\cdot j}\right) \mid \boldsymbol{X}=\boldsymbol{x} ; \theta\right) \equiv\left\{E\left(q_{q_{\cdot j}} \mid \boldsymbol{X}=\boldsymbol{x} ; \theta\right) \mid q_{q_{\cdot j}} \in \operatorname{Sel}\left(\mathbb{Q}_{j, \theta_{u}}\left(\boldsymbol{X}, \epsilon_{\cdot j}\right)\right)\right\}
$$

$\forall \boldsymbol{x} \in \mathscr{X}^{n}$. Hence, $\mathbb{E}\left(\mathbb{Q}_{j, \theta_{u}}\left(\boldsymbol{X}, \epsilon_{\cdot j}\right) \mid \boldsymbol{X}=\boldsymbol{x} ; \theta\right)$ is the collection of the probability distributions of $G$.j conditional on $\boldsymbol{X}=\boldsymbol{x}$ predicted by the model given $\theta$.

Therefore, $\Theta^{\star}$ can be equivalently expressed as

$$
\Theta^{\star}=\left\{\theta \in \Theta \mid \mathbb{P}(\boldsymbol{G} \mid \boldsymbol{X}=\boldsymbol{x}) \in \mathbb{E}\left(\mathbb{Q}_{\theta_{u}}(\boldsymbol{X}, \epsilon) \mid \boldsymbol{X}=\boldsymbol{x} ; \theta\right) \forall \boldsymbol{x} \in \mathscr{X}^{n} \text { a.s. }\right\},
$$

where $\mathbb{P}(\boldsymbol{G} \mid \boldsymbol{X}=\boldsymbol{x}) \equiv(\mathbb{P}(\boldsymbol{G}=\boldsymbol{g} \mid \boldsymbol{X}=\boldsymbol{x}) \forall \boldsymbol{g} \in \mathscr{G})$. Similarly, $\Theta^{\star \star}$ can be equivalently expressed as

$$
\Theta^{\star \star}=\left\{\theta \in \Theta \mid \mathbb{P}(G . j \mid \boldsymbol{X}=\boldsymbol{x}) \in \mathbb{E}\left(\mathbb{Q}_{j, \theta_{u}}(\boldsymbol{X}, \epsilon . j) \mid \boldsymbol{X}=\boldsymbol{x} ; \theta\right) \forall j \in \mathcal{N}, \forall \boldsymbol{x} \in \mathscr{X}^{n} \text { a.s. }\right\},
$$

where $\mathbb{P}\left(G_{\cdot j} \mid \boldsymbol{X}=\boldsymbol{x}\right) \equiv\left(\mathbb{P}\left(G_{\cdot j}=g_{\cdot j} \mid \boldsymbol{X}=\boldsymbol{x}\right) \forall g_{\cdot j} \in\{0,1\}^{n-1}\right)$.

Step 2.2 Given the selection $\boldsymbol{g} \equiv\left(g_{\cdot 1}, \ldots, g_{\cdot n}\right) \in \operatorname{Sel}\left(S_{\theta_{u}}(\boldsymbol{X}, \epsilon)\right)$ and the realisation $\boldsymbol{g} \equiv$ $\left(g_{\cdot 1}, \ldots, g_{\cdot n}\right) \in \mathscr{G}$, let $E\left(q_{q} \mid \boldsymbol{X}=\boldsymbol{x} ; \theta\right)_{\boldsymbol{g}}$ denote the component of the vector $E\left(q_{g} \mid \boldsymbol{X}=\boldsymbol{x} ; \theta\right)$ corresponding to $\boldsymbol{g}$. Let $E\left(\boldsymbol{q}_{q_{\cdot j}} \mid \boldsymbol{X}=\boldsymbol{x} ; \theta\right)_{g_{\cdot j}}$ denote the component of the vector $E\left(q_{q_{\cdot j}} \mid \boldsymbol{X}=\right.$ $\boldsymbol{x} ; \theta)$ corresponding to $g \cdot j$. Under Assumption 3 a),

$$
\begin{aligned}
& E\left(q_{g} \mid \boldsymbol{X}=\boldsymbol{x} ; \theta\right) \overbrace{\boldsymbol{g}}^{(C .5)} E\left(\Pi_{j=1}^{n} \mathbb{1}\left\{\epsilon_{\cdot j} \in \mathscr{E}_{\theta_{u}, q \cdot j}^{g_{\cdot j}}\right\} \mid \boldsymbol{X}=\boldsymbol{x} ; \theta\right) \boldsymbol{g} \overbrace{=}^{\text {Ass.3 a })} \Pi_{j=1}^{n} E\left(q_{q_{\cdot j}} \mid \boldsymbol{X}=\boldsymbol{x} ; \theta\right)_{g_{\cdot j}}, \\
& \forall \boldsymbol{g} \equiv\left(g_{\cdot 1}, \ldots, g \cdot n\right) \in \mathscr{G}, \forall \boldsymbol{x} \in \mathscr{X}^{n} \text {, and } \forall \theta \in \Theta .
\end{aligned}
$$


Step 2.3 Let $\theta^{0} \equiv\left(\theta_{u}^{0}, \theta_{\epsilon}^{0}\right)$ denote the true parameter value. Under Assumption 3,

$$
\begin{aligned}
& \mathbb{P}(\boldsymbol{G}=\boldsymbol{g} \mid \boldsymbol{X}=\boldsymbol{x}) \equiv \int_{e \in \mathbb{R}^{n(n-1)}} \mathbb{P}(\boldsymbol{G}=\boldsymbol{g} \mid \boldsymbol{X}=\boldsymbol{x}, \epsilon=e) d F\left(e ; \theta_{\epsilon}^{0}\right) \\
& \equiv \int_{e \equiv\left(e \cdot 1, \ldots, e_{\cdot n}\right) \in \mathbb{R}^{n(n-1)}} \mathbb{P}\left(G_{\cdot 1}=g_{\cdot 1}, \ldots, G_{\cdot n}=g_{\cdot n} \mid \boldsymbol{X}=\boldsymbol{x}, \epsilon \cdot 1=e_{\cdot 1}, \ldots, \epsilon_{\cdot n}=e \cdot n\right) d F\left(e ; \theta_{\epsilon}^{0}\right) \\
& \overbrace{=}^{\text {Ass.3 }} \Pi_{j=1}^{n} \int_{e \cdot j \in \mathbb{R}^{n-1}} \mathbb{P}\left(G_{\cdot j}=g_{\cdot j} \mid \boldsymbol{X}=\boldsymbol{x}, \epsilon_{\cdot j}=e_{\cdot j}\right) d F_{j}\left(e_{\cdot j} ; \theta_{\epsilon}^{0}\right) \\
& \equiv \Pi_{j=1}^{n} \mathbb{P}\left(G_{\cdot j}=g_{\cdot j} \mid \boldsymbol{X}=\boldsymbol{x}\right), \\
& \forall \boldsymbol{g} \equiv(g \cdot 1, \ldots, g \cdot n) \in \mathscr{G} \text { and } \forall \boldsymbol{x} \in \mathscr{X}^{n} .
\end{aligned}
$$

Step 2.4 By (C.7), if $\theta \in \Theta^{\star \star}$, then $\forall j \in \mathcal{N}$ there exists $\mathscr{q}_{\cdot j} \in \operatorname{Sel}\left(\mathcal{S}_{j, \theta_{u}}\left(\boldsymbol{X}, \epsilon_{\cdot j}\right)\right)$ such that

$$
\mathbb{P}\left(G_{\cdot j}=g_{\cdot j} \mid \boldsymbol{X}=\boldsymbol{x}\right)=E\left(q_{g_{\cdot j}} \mid \boldsymbol{X}=\boldsymbol{x} ; \theta\right)_{g_{\cdot j}},
$$

$\forall g_{\cdot j} \in\{0,1\}^{n-1}$ and $\forall \boldsymbol{x} \in X^{n}$ a.s. This implies

$$
\Pi_{j=1}^{n} \mathbb{P}\left(G_{\cdot j}=g_{\cdot j} \mid \boldsymbol{X}=\boldsymbol{x}\right)=\Pi_{j=1}^{n} E\left(q_{q_{\cdot j}} \mid \boldsymbol{X}=\boldsymbol{x} ; \theta\right)_{g_{\cdot j}},
$$

$\forall g_{\cdot j} \in\{0,1\}^{n-1}$ and $\forall \boldsymbol{x} \in X^{n}$ a.s., which, by steps 2.2 and 2.3 , is equivalent to

$$
\mathbb{P}(\boldsymbol{G}=\boldsymbol{g} \mid \boldsymbol{X}=\boldsymbol{x})=E\left(q_{g} \mid \boldsymbol{X}=\boldsymbol{x} ; \theta\right)_{\boldsymbol{g}},
$$

$\forall \boldsymbol{g} \equiv\left(g_{1}, \ldots, g_{\cdot n}\right) \in \mathscr{G}$ and $\forall \boldsymbol{x} \in \mathscr{X}^{n}$ a.s. Hence, by (C.6), $\theta \in \Theta^{\star}$.

\section{How to construct $\mathscr{A} g$}

We start with explaining how to construct $A_{g} g$ when $n=3$. First, note that

$$
\{0,1\}^{n-1}=\{(1,1),(1,0),(0,1),(0,0)\} .
$$

Hence,

$$
\begin{aligned}
\mathscr{K}_{\{0,1\}^{n-1}}=\{ & \{(1,1),(1,0),(0,1),(0,0)\},\{(1,1),(1,0)\},\{(1,1),(0,1)\},\{(1,1),(0,0)\},\{(1,0),(0,1)\}, \\
& \{(1,0),(0,0)\},\{(0,1),(0,0)\},\{(1,1),(1,0),(0,1)\},\{(1,1),(1,0),(0,0)\},\{(1,1),(0,1),(0,0)\}, \\
& \{(1,0),(0,1),(0,0)\},\{(1,1)\},\{(1,0)\},\{(0,1)\},\{(0,0)\}\} .
\end{aligned}
$$


$A_{g}$ is obtained by taking the Cartesian product of all the possible ordered $n$-tuples with repetitions from $\mathscr{K}_{\{0,1\}^{n-1}}$. For example, Alg contains

$$
\begin{aligned}
& \underbrace{\{(1,1)\}}_{\begin{array}{c}
\text { Set of equilibria } \\
\text { of section 1 game }
\end{array}} \times \underbrace{\{(1,1),(0,0)\}}_{\begin{array}{c}
\text { Set of equilibria } \\
\text { of section 2 game }
\end{array}} \times \underbrace{\{(0,0)\}}_{\begin{array}{c}
\text { Set of equilibria } \\
\text { of section } 3 \text { game }
\end{array}} \\
& =\{\{(1,1),(1,1),(0,0)\},\{(1,1),(0,0),(0,0)\}\} \underbrace{\left\{\left(\begin{array}{lll}
0 & 1 & 1 \\
1 & 0 & 1 \\
0 & 0 & 0
\end{array}\right),\left(\begin{array}{lll}
0 & 1 & 1 \\
0 & 0 & 0 \\
0 & 0 & 0
\end{array}\right)\right\}}_{\begin{array}{c}
\text { Rearrange } \\
\text { in matrix form } \\
\text { by inserting } \\
\text { a zero diagonal }
\end{array}} .
\end{aligned}
$$

Similarly, Alg contains

$$
\{(0,1)\} \times\{(0,1)\} \times\{(1,0)\}=\left\{\left(\begin{array}{lll}
0 & 0 & 1 \\
0 & 0 & 1 \\
1 & 0 & 0
\end{array}\right)\right\}
$$

By repeating such a calculation for all the possible ordered $n$-tuples with repetitions from $\mathscr{K}_{\{0,1\}^{n-1}}$, one finds that $\left|\mathscr{A}_{g}\right|=\left(2^{2^{n-1}}-1\right)^{n}=3375$.

Conversely, $A_{g}$ does to contain, e.g.,

$$
\left\{\left(\begin{array}{lll}
0 & 1 & 1 \\
1 & 0 & 0 \\
0 & 0 & 0
\end{array}\right),\left(\begin{array}{lll}
0 & 0 & 0 \\
0 & 0 & 0 \\
0 & 0 & 0
\end{array}\right),\left(\begin{array}{lll}
0 & 1 & 0 \\
0 & 0 & 1 \\
1 & 1 & 0
\end{array}\right)\right\},
$$

and

$$
\left\{\left(\begin{array}{lll}
0 & 1 & 1 \\
1 & 0 & 0 \\
0 & 0 & 0
\end{array}\right),\left(\begin{array}{lll}
0 & 0 & 0 \\
1 & 0 & 0 \\
0 & 0 & 0
\end{array}\right),\left(\begin{array}{lll}
0 & 1 & 1 \\
1 & 0 & 0 \\
0 & 1 & 0
\end{array}\right)\right\} .
$$

We now generalise the procedure to any $n$. Consider $n$ sets, $K_{1} \in \mathscr{K}_{\{0,1\}^{n-1}}, \ldots, K_{n} \in$ $\mathscr{K}_{\{0,1\}^{n-1}}$. Construct the set $\mathscr{B}_{K_{1}, \ldots, K_{n}} \equiv X_{j=1}^{n} K_{j}$. Hence, $\mathscr{B}_{K_{1}, \ldots, K_{n}}$ is a collection of $L \equiv$ $\Pi_{j=1}^{n}\left|K_{j}\right|$ sets and it can be written as $\left\{B_{l}\right\}_{l=1}^{L}$. Any set $B_{l} \in \mathscr{B}_{K_{1}, \ldots, K_{n}}$ is composed by $n$ vectors of size $(n-1) \times 1$. Thus, $B_{l} \equiv\left\{b_{l, 1}, \ldots, b_{l, n}\right\}$ with $b_{l, h} \equiv\left(b_{l, h}^{1}, \ldots, b_{l, h}^{n-1}\right) \forall h \in\{1, \ldots, n\}$, $\forall l \in\{1, \ldots, L\}$. Create the $n \times n$ matrix

$$
C_{l} \equiv\left(\begin{array}{ccccccc}
0 & b_{l, 2}^{1} & b_{l, 3}^{1} & \ldots & b_{l, h}^{1} & \ldots & b_{l, n}^{1} \\
b_{l, 1}^{1} & 0 & b_{l, 3}^{2} & \ldots & b_{l, h}^{2} & \ldots & b_{l, n}^{2} \\
b_{l, 1}^{2} & b_{l, 2}^{2} & 0 & \ldots & b_{l, h}^{3} & \ldots & b_{l, n}^{3} \\
\vdots & \vdots & \vdots & \ldots: \vdots & \vdots & \ldots: . \vdots & \vdots \\
b_{l, 1}^{n-2} & b_{l, 2}^{n-2} & b_{l, 3}^{n-2} & \ldots & b_{l, h}^{n-2} & \ldots & b_{l, n}^{n-1} \\
b_{l, 1}^{n-1} & b_{l, 2}^{n-1} & b_{l, 3}^{n-1} & \ldots & b_{l, h}^{n-1} & \ldots & 0
\end{array}\right)
$$

$\forall l \in\{1, \ldots, L\} . \quad$ Let $A \equiv\left\{C_{1}, \ldots, C_{L}\right\}$. Repeat the procedure for all the possible $K_{1} \in$

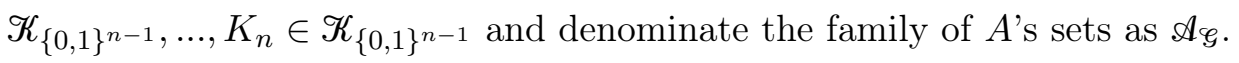




\section{References}

Aldrich, H.E. (1979): Organizations and Environments, Englewood Cliffs, NJ: Prentice-Hall.

Allen, M.P. (1974): "The Structure of Interorganizational Elite Cooptation: Interlocking Corporate Directorates", American Sociological Review, 39(3), 393-406.

Andrews, D.W.K., and G. Soares (2010): "Inference for Parameters Defined by Moment Inequalities Using Generalized Moment Selection", Econometrica, 78(1), 119-157.

Aradillas-Lopez, A., and A.M. Rosen (2016): "Inference in Ordered Response Games with Complete Information", Working Paper.

Bajari, P., H. Hong, and S.P. Ryan (2010): "Identification and Estimation of a Discrete Game of Complete Information", Econometrica, 78(5), 1529-1568.

Badev, A. (2017): "Discrete Games in Endogenous Networks: Equilibria and Policy", Working Paper arXiv:1705.03137.

Barbi, V. (2000): "Interlocking Directorhip Networks: What is Relevant for the Evolution and Change of the Networks?", Quaderni del Dipartimento di Economia Politica, 278, Universitá di Siena.

Baysinger, B.D., and H.N. Butler (1985): "Corporate Governance and the Board of Directors: Performance Effects of Changes in Board Composition", Journal of Law, Economics, \& Organization, 1(1), 101-124.

Bellenzier, L., and R. Grassi (2014): "Interlocking Directorates in Italy: Persistent Links in Network Dynamics", Journal of Economic Interaction and Coordination, 9(2), 183-202.

Beresteanu, A., I. Molchanov, and F. Molinari (2011): "Sharp Identification in Models with Convex Moment Predictions", Econometrica, 79(6), 1785-1821.

Berry, S. (1992): "Estimation of a Model of Entry in the Airline Industry", Econometrica, 60(4), 889-917.

Bertoni, F., and P. Randone (2006): "The Small-World of Italian Finance: Ownership Interconnections and Board Interlocks amongst Italian Listed Companies", SSR Working Paper.

Bianco, M., and E. Pagnoni (1997): "Board Interlocks across Listed Companies in Italy: the Case of Banks", BNL Quarterly Review, Special Issue on Property, Control and Corporate Governance of Banks.

Bloch, F., and M. Jackson (2005): "The Formation of Networks with Transfers among Players", Journal of Economic Theory, 133(1), 83-110.

Bloch, F., and M. Jackson (2006): "Definitions of Equilibrium in Network Formation Games", International Journal of Game Theory, 34(3), 305-318. 
Bontemps, C., and R. Kumar (2018): "A Geometric Approach to Inference in Set-Identified Entry Games", TSE Working Paper 943.

Booth, J.R., and D.N. Deli (1996): "Factors Affecting the Number of Outside Directorships Held by CEOs", Journal of Financial Economics, 40(1), 81-104.

Boucher, V. (2017): "The Estimation of Network Formation Games with Positive Spillovers", CRREP Working Paper Series 2017-10.

Bunting, D. (1976): "Corporate Interlocking. Part III. Interlocks and Return on Investment", Directors \& Boards, 1(3), 4-11.

Burt, R.S. (1983): Corporate Profits and Cooptation, New York: Academic.

Cai, J., and A. Szeidl (2018): "Interfirm Relationships and Business Performance", Quarterly Journal of Economics, 133(3), 1229-1282.

Calvó-Armengol, A. (2004): "Job Contact Networks", Journal of Economic Theory, 115(1), 191-206.

Calvó-Armengol, A., and M.O. Jackson (2004): "The Effects of Social Networks on Employment and Inequality", American Economic Review, 94(3), 426-454.

Calvó-Armengol, A., and Y. Zenou (2004): "Social Networks and Crime Decisions: The Role of Social Structure in Facilitating Delinquent Behavior", International Economic Review, 45(3), 939-958.

Calvó-Armengol, A., and R. Ilkiliç (2009): "Pairwise-Stability and Nash Equilibria in Network Formation", International Journal of Game Theory, 38(1), 51-79.

Carrington, P.J. (1981): Horizontal Co-optation through Corporate Interlocks, PhD thesis, Dept. Sociol. Univ. Toronto.

Chesher, A., and A.M. Rosen (2019): "Modelling Simultaneously Determined Discrete Outcomes with Application to Firm Entry and Product Quality Choice", Working Paper.

Christakis, N., J. Fowler, G. Imbens, and K. Kalianaraman (2010): "An Empirical Model of Strategic Network Formation", NBER Working Paper 16039.

Ciliberto, F., and E. Tamer (2009): "Market Structure and Multiple Equilibria in Airline Markets", Econometrica, 77(6), 1791-1828.

Ciocca, P. (2007): Ricchi per Sempre? Una Storia Economica d'Italia (1796-2005). Torino, Bollati-Boringhieri.

Conley, T.G., and C.R. Udry (2010): "Learning about a New Technology: Pineapple in Ghana", American Economic Review, 100(1), 35-69.

Core, J.E, R.W. Holthausen, D.F. Larcker (1999): "Corporate Governance, Chief Executive Officer Compensation, and Firm Performance", Journal of Financial Economics, 51(3), 371-406. 
Currarini, S., M. Jackson, and P. Pin (2009): "An Economic Model of Friendship: Homophily, Minorities and Segregation", Econometrica, 77(4), 1003-1045.

Dellarocas, C., Z. Katona, and W. Rand (2013): "Media, Aggregators, and the Link Economy: Strategic Hyperlink Formation in Content Networks", Management Science, 59(10), 23602379 .

de Paula, A. (2013): "Econometric Analysis of Games with Multiple Equilibria", Annual Review of Economics, 5(1), 107-131.

de Paula, A. (2017): "Econometrics of Network Models", in Advances in Economics and Econometrics: Theory and Applications: Eleventh World Congress (Econometric Society Monographs), ed. by B. Honore, A. Pakes, M. Piazzesi and L. Samuelson, Cambridge: Cambridge University Press.

de Paula, A., S. Richards-Shubik, and E. Tamer (2018): "Identifying Preferences in Networks with Bounded Degree", Econometrica, 86(1), 263-288.

Dooley, P.C. (1969): "The Interlocking Directorate", American Economic Review, 59(3), 314323.

Fligstein, N., and P. Brantley (1992): "Bank Control, Owner Control, or Organizational Dynamics: Who Controls the Large Modern Corporation?", American Journal of Sociology, 98(2), 280-307.

Fukumizu, K., A. Gretton, X. Sun, and B. Schölkopf (2008): "Kernel Measures of Conditional Dependence", in Advances in Neural Information Processing Systems 20 (NIPS), MIT Press, 489-496.

Gabrielsen, T.S., E. Hjelmeng, and L. Sørgard (2011): "Rethinking Minority Share Ownership and Interlocking Directorships: The Scope for Competition Law Intervention", European Law Review, 6, 837-860.

Galichon, A., and M. Henry (2006): "Inference in Incomplete Models", SSR Working paper.

Galichon, A., and M. Henry (2011): "Set Identification in Models with Multiple Equilibria", Review of Economic Studies, 78(4), 1264-1298.

Gayle, G.-L., L. Golan, and R.A. Miller (2015): "Interlocked Executives and Insider Board Members: An Empirical Analysis", Federal Reserve Bank of St. Louis Working Paper 2015-040A.

Gilles, R.P., and S. Sarangi (2005): "Stable Networks and Convex Payoff", Working Paper.

Goldsmith-Pinkham, and G.W. Imbens (2013): "Social Networks and the Identification of Peer Effects", Journal of Business \& Economic Statistics, 31(3), 253-264.

Goyal, S., and S. Joshi (2006): "Unequal links", International Journal of Game Theory, 34(3), 319-349. 
Graham, B. (2015): "Methods of Identification in Social Networks", Annual Review of Economics, 7, 465-485.

Hallock, K.F. (1997): "Reciprocally Interlocking Boards of Directors and Executive Compensation", Journal of Financial and Quantitative Analysis, 32(3), 331-344.

Helmers, C., M. Patnam, and P.R. Rau (2015): "Do Board Interlocks Increase Innovation? Evidence from a Corporate Governance Reform in India", SSR Working Paper.

Hsieh, C.S., and L.F. Lee (2016): "A Social Interactions Model with Endogenous Friendship Formation and Selectivity", Journal of Applied Econometrics, 31(2), 301-319.

Jackson, M.O. and J. Wolinsky (1996): "A Strategic Model of Social and Economic Networks", Journal of Economic Theory, 71(1), 44-74.

Kallenberg, O. (2005): Probabilistic Symmetries and Invariance Principles, Springer.

Kaplan, S.N., and D. Reishus (1990): "Outside Directorships and Corporate Performance", Journal of Financial Economics, 27(2), 389-410.

Kranton, R.E., and D.F. Minehart (2000a): "Networks versus Vertical Integration", Rand Journal of Economics, 31(3), 570-601.

Kranton, R.E., and D.F. Minehart (2000b): "Competition for Goods in Buyer-Seller Networks", Review of Economic Design, 5(3), 301-331.

Kranton, R.E., and D.F. Minehart (2001): "A Theory of Buyer-Seller Networks", American Economic Review, 91(3), 485-508.

Lalanne, M. (2018): "Social Networks and Job Referrals in Recruitment", Working Paper.

Lazega, E., C. Lemercier, and L. Mounier (2006): "A Spinning Top Model of Formal Structure and Informal Behaviour: Dynamics of Advice Networks in a Commercial Court", European Management Review, 3(2), 113-122.

Lazega, E., L. Mounier, T. Snijders, and P. Tubaro (2012): "Norms, Status and the Dynamics of Advice Networks: a Case Study", Social Networks, 34(3), 323-332.

Lazega, E., and T. Snijders (2015): Multilevel Network Analysis for the Social Sciences: Theory, Methods and Applications. Springer.

Leslie, C. (2004): "Trust, Distrust, and Antitrust", Texas Law Review, 83(3), 515-680.

Leung, M. (2015): "Two-Step Estimation of Network Formation Models with Incomplete Information", Journal of Econometrics, 188(1), 182-195.

Leung, M. (2019): "A Weak Law for Moments of Pairwise-Stable Networks", Journal of Econometrics, 210(2), 310-326.

Luzzatto Fegiz, P. (1928): "Il Consiglio d'Amministrazione e l'Interdipendenza delle Imprese", Giornale degli Economisti e Rivista di Statistica, 68(3), 197-231. 
McFadden, D. (1989): "A Method of Simulated Moments for Estimation of Discrete Response Models without Numerical Integration", Econometrica, 57(2), 995-1026.

Meeusen, W., and L. Cuyvers (1985): "The Interaction between Interlocking Directorships and the Economic Behaviour of Companies", in Networks of Corporate Power, ed. by N. Stokman, R. Ziegler, and J. Scott, Cambridge, England: Polity.

Mele, A. (2017): "A Structural Model of Dense Network Formation", Econometrica, 85(3), $825-850$.

Menzel, K. (2016): "Strategic Network Formation with Many Agents", Working Paper.

Mintz, B., and M. Schwartz (1981): "The Structure of Intercorporate Unity in American Business", Social Problems, 29(2), 87-103.

Miyauchi, Y. (2016): "Structural Estimation of Pairwise Stable Networks with Nonnegative Externality", Journal of Econometrics, 195(2), 224-235.

Mizruchi, M.S. (1996): "What Do Interlocks Do? An Analysis, Critique, and Assessment of Research on Board Interlocks", Annual Review of Sociology, 22, 271-298.

Mizruchi, M.S, and D. Bunting (1981): "Influence in Corporate Networks: an Examination of Four Measures", Administrative Science Quarterly, 26(3), 475-489.

Mizruchi, M.S., and L.B. Stearns (1988): "A Longitudinal Study of the Formation of board interlocks", Administrative Science Quarterly, 33(2), 194-210.

Mizruchi, M.S., and L.B. Stearns (1994): "A Longitudinal Study of Borrowing by Large American Corporations", Administrative Science Quarterly, 39(1), 118-140.

Nakajima, R. (2007): "Measuring Peer Effects on Youth Smoking Behaviour", Review of Economic Studies, 74(3), 897-935.

OECD (2008): Antitrust Issues Involving Minority Shareholding and Interlocking Directorates, available here.

OECD (2010): Information Exchanges Between Competitors Under Competition Law, available here.

Pakes, A., and D. Pollard (1989): "Simulation and the Asymptotics of Optimization Estimators", Econometrica, 57(5), 1027-1057.

Patnam, M. (2013): "Corporate Networks and Peer Effects in Firm Policies", Working Paper.

Pennings, J.M. (1980): Board Interlocks, San Francisco, Calif.: Jossey-Bass.

Pfeffer J. (1972): "Size and Composition of Corporate Boards of Directors: The Organization and its Environment", Administrative Science Quarterly, 17(2), 218-228.

Pfeffer J., and G.R. Salancik (1978): The External Control of Organisations: A Resource Dependence Perspective, New York: Harper \& Row. 
Ridder, G., and S. Sheng (2017): "Estimating large network formation games", Working Paper.

Rinaldi, A., and M. Vasta (2005): "The Structure of Italian Capitalism, 1952-1972: New Evidence Using the Interlocking Directorates Technique", Financial History Review, 12(2), 173-198.

Rinaldi, A., and M. Vasta (2012): "The Italian Corporate Network After the Golden Age (1972-1983): from Centrality to Marginalization of State-Owned Enterprises", Enterprise and Society, 13(2), 378-413.

Sacerdote, B. (2001): "Peer Effects with Random Assignment: Results for Dartmouth Roommates", Quarterly Journal of Economics, 116(2), 681-704.

Santella, P., C. Drago, and A. Polo (2009): "The Italian Chamber of Lords Sits on Listed Company Boards: An Empirical Analysis of Italian Listed Company Boards from 1998 to 2006", SSR Working Paper.

Santella, P., C. Drago, A. Polo, and E. Gagliardi (2009): "A Comparison Among the Director Networks in the Main Listed Companies in France, Germany, Italy, and the United Kingdom", MPRA Working Paper 16397.

Selznick, P. (1949): TVA and the Grass Roots, New York: Harper \& Row.

Sheng, S. (2016): "A Structural Econometric Analysis of Network Formation Games", Working Paper.

Stokman, F.N, J. Van Der Knoop, and F.W. Wasseur (1988): "Interlocks in the Netherlands: Stability and Careers in the Period 1960-1980", Social Networks, 10(2), 183-208.

Stokman, F.N., F.W. Wasseur, and D. Elsas (1985): "The Dutch Network. Types of Interlocks and Network Structure", in Networks of Corporate Power. A comparative Analysis of Ten Countries, ed. by R. Ziegler, and J. Scott, Oxford: Polity Press.

Tamer, E. (2003): "Incomplete Simultaneous Discrete Response Model with Multiple Equilibria", Review of Economic Studies, 70(1), 147-167.

Thompson, J.D., and W. McEwen (1958): "Organizational Goals and Environment: GoalSetting as an Interaction Process", American Sociological Review, 23(1), 23-31.

Topkis, D.M (1979): "Equilibrium Points in Nonzero-Sum n-Person Submodular Games", Journal of Control and Optimization, 17(6), 773-787.

Torgovitsky, A. (2018): "Partial Identification by Extending Subdistributions", Quantitative Economics, forthcoming.

Vasta, M., and A. Baccini (1997): "Banks and Industry in Italy, 1911-36: New Evidence Using the Interlocking Directorates Technique", Financial History Review, 4(2), 139-159.

Waller, S.W. (2011): "Corporate Governance and Competition Policy", George Mason Law Review, 18(4), 833-887. 
Zhang, K., J. Peters, D. Janzing, and B. Schölkopf (2011): "Kernel-Based Conditional Independence Test and Application in Causal Discovery", in Proceedings of the 27th Annual Conference on Uncertainty in Artificial Intelligence (UAI), AUAI Press, 804-813. 